\title{
1 Simultaneous infection with porcine reproductive and respiratory 2 syndrome and influenza viruses abrogates clinical protection 3 induced by live attenuated porcine reproductive and respiratory 4 syndrome vaccination
}

5 Tiphany Chrun $^{1 *}$, Emmanuel A. Maze ${ }^{1}$, Eleni Vatzia ${ }^{1}$, Veronica Martini ${ }^{1}$, Basu 6 Paudyal $^{1}$, Matthew Edmans ${ }^{1}$, Adam McNee $^{1}$, Tanuja Manjegowda ${ }^{1}$, Francisco J. 7 Salguero $^{2}$, Nanchaya Wanasen ${ }^{3}$, Surapong Koonpaew ${ }^{3}$, Simon P. Graham ${ }^{1 * \dagger}$ and 8 Elma Tchilian ${ }^{1 * \dagger}$

$9 \quad{ }^{1}$ The Pirbright Institute, Woking, United Kingdom

$10{ }^{2}$ Public Health England, Salisbury, United Kingdom

$11{ }^{3}$ Virology and Cell Technology Laboratory, National Center for Genetic

12 Engineering and Biotechnology (BIOTEC), National Science and Technology

13 Development Agency, Pathum Thani, Thailand

$14 *$ Correspondence:

15 Tiphany Chrun tiphany.chrun@ pirbright.ac.uk

16 Simon Graham simon.graham@ pirbright.ac.uk

17 Elma Tchilian elma.tchilian@pirbright.ac.uk

$18 †$ These authors have contributed equally to this work.

19 Keywords: Porcine reproductive and respiratory syndrome virus, swine influenza A 20 virus, live attenuated vaccine, pig, co-infection. 


\section{Abstract}

23 The porcine respiratory disease complex (PRDC) is responsible for significant

24 economic losses in the pig industry worldwide. Porcine reproductive and respiratory

25 syndrome virus (PRRSV) and swine influenza virus are major viral contributors to

26 PRDC. Vaccines are cost-effective measures for controlling PRRS, however, their

27 efficacy in the context of co-infections has been poorly investigated. In this study,

28 we aimed to determine the effect of PRRSV-2 and swine influenza H3N2 virus co-

29 infection on the efficacy of PRRSV modified live virus (MLV) vaccination, which is

30 widely used in the field. Following simultaneous challenge with contemporary

31 PRRSV-2 and H3N2 field isolates, we found that the protective effect of PRRS

32 MLV vaccination on clinical disease and pathology was abrogated, although viral

33 load was unaffected and antibody responses were enhanced. In contrast, co-infection

34 in non-immunized animals reduced PRRSV-2 viremia and H3N2 virus load in the

35 upper respiratory tract and potentiated T cell responses against both PRRSV-2 and

$36 \mathrm{H} 3 \mathrm{~N} 2$ in the lung. Further analysis suggested that an upregulation of inhibitory

37 cytokines gene expression in the lungs of vaccinated pigs may have influenced

38 responses to $\mathrm{H} 3 \mathrm{~N} 2$ and PRRSV-2. These findings provide important insights into

39 the effect of viral co-infections on PRRS vaccine efficacy that may help identity

40 more effective vaccination strategies against PRDC in the field. 


\section{Introduction}

44 Porcine reproductive and respiratory syndrome (PRRS) is a viral disease responsible

45 for major economic losses in the global pig industry (1). The disease can be

46 subclinical depending on the strain $(2,3)$, however typical clinical signs are

47 reproductive failure in sows, respiratory distress and reduction of growth

48 performance in weaned and growing pigs (4). Mortality can be observed in infected

49 piglets, with rates ranging from $7.5-18.5 \%$ (5) and in some cases up to $75.5 \%$ (6).

50 The etiologic agents are PRRS viruses (PRRSV), single stranded positive RNA

51 viruses from the Arteriviridae family (7). The first clinical description of PRRS

52 dates to the late 1980's, with genetically distinct PRRSV isolates described in

53 Europe and North America, which are now recognized as two separate species

54 PRRSV-1 (Betaarterivirus suid 1) and -2 (Betaarterivirus suid 2), respectively (7).

55 Both species have since spread globally, but PRRSV-1 remains predominant in

56 Europe, while PRRSV-2 predominates in the Americas and Asia. The rapid

57 evolution of PRRSV due to a high mutation rate when replicating its genome, and

58 recombination between strains have resulted in substantial genetic diversity, which

59 poses challenges for the control of PRRS by vaccination (8).

60

Vaccination is widely practiced as one of measures used to prevent and

61 control PRRS. Commercially available vaccines include inactivated and live

62 attenuated (also known as modified live virus; MLV) vaccines for both PRRSV-1

63 and -2 (9). Although inactivated vaccines are considered safer, MLVs are

64 preferentially used for their higher protective efficacy $(8,10)$. Studies have

65 demonstrated an induction of a high level of PRRSV-specific antibodies which is

66 associated with clinical protection against challenge infection with related PRRSV

67 strains (9), but rather weak T cell responses after MLV immunization (11). Passive

68 transfer of purified IgG from PRRS-convalescent pigs have suggested that vaccine-

69 induced protection against reproductive failure and vertical transmission in utero is

70 also mediated by antibodies (12,13). Since PRRS MLV vaccine-induced antibodies

71 often lack strong virus neutralizing properties in vitro (14), non-neutralizing

72 antibody functions may also contribute but these remain poorly defined (15). 
73 Multiple infections of pigs tend to occur naturally in the field (reviewed in 74 (16)). Indeed, respiratory diseases in pigs in the field are often multifactorial, 75 involving mixed infections with different viral and/or bacterial pathogens, defined as 76 the porcine respiratory disease complex (PRDC). PRRSV and swine influenza A 77 virus (swIAV), an enveloped single stranded segmented negative RNA virus within 78 the Orthomyxoviridae family (7), are important contributors to the PRDC. Primary 79 infection with PRRSV and swIAV leads to pneumonia caused by opportunistic 80 pathogens, such as Pasteurella multocida, Mycoplasma hyopneumonia, 81 Actinobacillus pleuropneumoniae and Bordetella bronchiseptica (16). Previous in 82 vivo PRRSV/swIAV co-infection or superinfections studies demonstrated a 83 potentiation of disease compared to single infection $(17,18)$. It was recently shown 84 that concomitant swIAV infection modulated the immune response to PRRS MLV 85 vaccination, albeit without impacting efficacy (19), but it remains unknown whether 86 such viral co-infection interferes with protection conferred by PRRS vaccination.

88 PRRSV-2/H3N2 co-infection. We evaluated clinical signs, viral load, PRRSV-289 specific antibody and T cell responses in PRRS MLV-vaccinated pigs challenged 90 simultaneously with contemporary field-isolated PRRSV-2 and swIAV H3N2

91 strains. We report here that PRRSV-2/H3N2 co-infection abrogated the protective 92 effect of PRRS MLV vaccine on lung pathology, although it did not alter viral load.

93 Moreover, PRRS MLV reversed the beneficial effect of PRRSV-2/H3N2 co94 infection in decreasing H3N2 lung viral loads, highlighting a potential interference 95 of PRRS MLV vaccination on the subsequent host immune response against H3N2. 
97

98

99

100

101

102

103

104

105

106

107

108

109

110

111

112

113

114

115

116

117

118

119

120

121

122

123

124

125

126

127

Materials and methods

\section{Cell lines}

Madin-Darby canine kidney (MDCK) cells were cultured in Eagle's minimum essential medium (MEM, Merck, Feltham, UK) supplemented with $10 \%$ heatinactivated fetal bovine serum (HI FBS, Thermo Fisher Scientific, Loughborough, UK) and antibiotics (100 U/mL penicillin and $100 \mu \mathrm{g} / \mathrm{mL}$ streptomycin, Thermo Fisher) at $37{ }^{\circ} \mathrm{C}$ in a humidified $5 \% \mathrm{CO}_{2}$. African green monkey kidney (MARC145) cells were cultured in Dulbecco's modified MEM (DMEM, Merck) supplemented with $10 \% \mathrm{HI} \mathrm{FBS}$ and antibiotics at $37{ }^{\circ} \mathrm{C}$ in a humidified $5 \% \mathrm{CO}_{2}$ atmosphere.

\section{Vaccine and virus strains}

PRRSV-2 16CB02 was isolated from pig serum collected from a diseased farm in Chonburi, Thailand in 2016. The serum was adsorbed onto MARC-145 cells and cultured in OptiMEM (Thermo Fisher Scientific) supplemented with $10 \%$ FBS. After $72 \mathrm{~h}$, cytopathic effect (CPE) was observed, and the supernatant was collected. Cells were then infected with a diluted culture supernatant before being overlayed with MEM supplemented with $0.3 \%$ bovine serum albumin (BSA, Merck), $0.22 \%$ sodium bicarbonate (Merck), $1 \%$ penicillin/streptomycin (Merck) and $1 \%$ methylcellulose (Merck). The PRRSV-2 16CB02 isolate (referred to as PRRSV-2) was derived from a single plaque-derived culture, which resulted in obvious CPE after $96 \mathrm{~h}$ incubation. Partial genome (ORFs 2-7) sequencing was performed (GenBank, accession number MZ700336) and the ORF5 sequence was compared with sequences in the NCBI database using BLAST showed that $16 \mathrm{CB} 02$ belonged to lineage 8.7 of PRRSV-2 (Betaarterivirus suid 2). PRRSV-2 16CB02 was propagated in MARC-145 cells by infection with a multiplicity of infection (MOI) of 0.08 for $1 \mathrm{~h}$ at $37{ }^{\circ} \mathrm{C}$ in $5 \% \mathrm{CO}_{2}$. After removing the inoculum cells were incubated for 3 days in DMEM with $10 \% \mathrm{FBS}$ and antibiotics at $37{ }^{\circ} \mathrm{C}$ in $5 \% \mathrm{CO}_{2}$. Supernatant was harvested, centrifuged at $500 \times \mathrm{g}$ for $10 \mathrm{~min}$ and stored at $-80{ }^{\circ} \mathrm{C}$ until use. The viral stock was titrated and expressed as TCID $50 / \mathrm{mL}$.

SwIAV H3N2 CM5 strain was isolated from a pig farm in the Province of Lumpoon, Thailand in 2018. Nasal swab samples were first screened for IAV by 
128 one-step RT-PCR. Viral RNA was extracted from samples with the GenUPTM Virus

129 RNA kit (biotechrabbit, Henningsdorf, Germany). One-step RT-PCR was conducted

130 using matrix (M) segment specific primers (20) and performed on a $\mathrm{T} 100^{\mathrm{TM}}$

131 Thermal Cycler (Bio-Rad) utilizing the PrimeScript ${ }^{\mathrm{TM}}$ One-Step RT-PCR kit

132 (Takara Bio, Shiga, Japan). PCR products were then separated and visualized by

133 agarose gel electrophoresis. The RT-PCR-positive nasal swab samples were then

134 subjected to IAV isolation using MDCK cells. CPE was observed daily, and CPE

135 positive supernatants collected and confirmed by HA test and RT-PCR for M gene

136 detection as previously described (21). Sequencing of H3N2 RNA segments were

137 performed and alignment of HA (GenBank, accession number MZ665044) and NA

138 (GenBank, accession number MZ665046) sequences against sequences in the NCBI

139 database revealed that CM5 strain was a H3N2 subtype. H3N2 CM5 (referred to as

$140 \mathrm{H} 3 \mathrm{~N} 2$ ) was propagated by infecting MDCK cells at MOI 0.001 in serum-free MEM

141 and incubated at $37{ }^{\circ} \mathrm{C}$ in $5 \% \mathrm{CO}_{2}$ for $1 \mathrm{~h}$. After washing the cell monolayer, cells

142 were incubated in serum-free MEM supplemented with $2 \mu \mathrm{g} / \mathrm{mL}$ TPCK-treated

143 trypsin (Merck) for 2 days at $37{ }^{\circ} \mathrm{C}$ in $5 \% \mathrm{CO}_{2}$. Supernatant was harvested,

144 centrifuged at $880 \times g$ for $10 \mathrm{~min}$ and stored at $-80{ }^{\circ} \mathrm{C}$ until use. The viral stock was

145 titrated by plaque assay and expressed as $\mathrm{pfu} / \mathrm{mL}$.

146 PRRS MLV (Ingelvac® PRRS MLV, Boehringer Ingelheim Vetmedica

$147 \mathrm{GmbH}$, Ingelheim am Rhein, Germany) was used following reconstitution according

148 to manufacturer's instructions.

\section{Immunization and challenge study}

150 The animal experiment was approved by the Animal Welfare and Ethical Review

151 Body at The Pirbright Institute, UK. The treatment, housing, husbandry, and

152 procedures were performed in accordance with the UK Animal (Scientific

153 Procedures) Act 1986 (Project Licence P6F09D691). Thirty-six, 5-7 weeks-old,

154 Large White-Landrace-Hampshire crossbred female pigs were sourced from a high

155 health status commercial herd and were housed in a high biocontainment facility at

156 The Pirbright Institute. Animals were tested for the absence of exposure to IAV and

157 PRRSV prior to their arrival by serological tests via hemagglutination inhibition test

158 against four standard IAV antigens (pdmH1N1, H1N2, H3N2 and avian-like H1N1 
159 strains), and antibody (Ab) ELISA and RT-PCR tests against PRRSV (Animal and

160 Plant Health Agency, Weybridge, UK). Pigs were randomly assigned to 6 groups of

1616 pigs each, which were untreated (naïve), immunized with $2 \mathrm{~mL}$ containing $10^{4.5}$

162 TCID $_{50}$ of Ingelvac® PRRS MLV (Vac) or with $2 \mathrm{~mL}$ of PBS (Ctrl) by

163 intramuscular (i.m.) injection (Figure 1A). On day 33 post-vaccination (dpv), Ctrl

164 and Vac pigs were inoculated intranasally with $4 \mathrm{~mL}(2 \mathrm{~mL} /$ nostril $)$ containing $5 \times$

$16510^{6}$ pfu of swIAV H3N2 CM5 (Ctrl + H3N2), $10^{5}$ TCID 50 PRRSV-2 16CB02 (Ctrl +

166 PRRSV-2 or Vac + PRRSV-2), or concurrently with $5 \times 10^{6}$ pfu swIAV and $10^{5}$

167 TCID 50 PRRSV-2 (Ctrl + PRRSV-2/H3N2 or Vac + PRRSV-2/swIAV) diluted in

168 DMEM using a mucosal atomization device (MAD 300, Wolfe Tory Medical, Salt

169 Lake City, USA). To analyze the immune response, pigs from Ctrl + PRRSV-2, Vac

$170+$ PRRSV-2 and Vac + PRRSV-2/H3N2 groups were bled at $0 \mathrm{dpv}$, then pigs from

171 all groups at 20 and $38 \mathrm{dpv}$ for peripheral blood mononuclear cell (PBMC) and

172 serum isolation. After the challenge, pigs were observed twice per day until the end

173 of the study for monitoring and scoring of clinical signs (Supplementary Table

174 S1). Rectal temperatures were taken on $-1,0,1,2,3$ and 4 days post-challenge

175 (dpc). Nasal swabs were collected daily for viral detection. At $5 \mathrm{dpc}$, the animals

176 were humanely euthanized with an overdose of pentobarbital sodium anesthetic.

\section{Pathological and histopathological examination of lungs}

178 Lungs were removed post-mortem and tissue samples were taken from cranial, 179 cardiac, and diaphragmatic lobes of the left lung, and immersed in $10 \%$ neutral 180 buffered formalin for fixation and histological processing. Formalin fixed tissues 181 were paraffin wax-embedded and $4 \mu \mathrm{m}$ sections cut and stained with hematoxylin 182 and eosin (H\&E). Immunohistochemical staining of IAV nucleoprotein (NP) and 183 PRRSV nucleoprotein $(\mathrm{N})$ protein was performed in $4 \mu \mathrm{m}$ tissue sections as 184 previously described $(22,23)$. Histopathological changes in stained lung tissue 185 sections were scored by a board-certified veterinary pathologist blinded to the 186 treatment group. Lung histopathology was scored ("Morgan score") using five 187 parameters (necrosis of the bronchiolar epithelium, airway inflammation, 188 perivascular/bronchiolar cuffing, alveolar exudates, and septal inflammation) scored 189 on a 5-point scale of 0 to 4 and then summed to give a total slide score ranging from 1900 to 20 and a total lung score from 0 to 60 (24). Two additional scoring systems 
191 were used to analyze the contribution of IAV ("Iowa") and PRRSV-2 ("Salguero")

192 in the lung lesions. Abundance of viral antigen was assessed using the influenza NP

193 staining (HB-65 mAb, Bio X Cell, Lebanon, USA) and was scored as described

194 previously (25). Similarly, PRRSV N protein staining was performed (SDOW17-A

195 mAb, Rural Technologies, Brookings, USA) and the scoring method was adapted

196 from the Iowa IAV scoring method as described above.

\section{Sample collection and cell isolation}

198 Blood samples were collected at 0, 20 and $38 \mathrm{dpv}$ using BD Vacutainer ${ }^{\mathrm{TM}}$ SST $^{\mathrm{TM}}$ II

199 Advance Tubes and BD Vacutainer Heparin Blood Collection Tubes (both Fisher

200 Scientific). Serum tubes were centrifuged at $880 \times g$ for $10 \mathrm{~min}$ and the resulting

201 serum was aliquoted and stored at $-80{ }^{\circ} \mathrm{C}$. Heparinized blood diluted 1:1 in PBS

202 overlaid onto Lymphopure density gradient medium 1.077 g/mL (BioLegend, San

203 Diego, USA) in Leucosep tubes (Greiner Bio-One, Gloucestershire, UK) and

204 centrifuged at $800 \times g$ for $15 \mathrm{~min}$. PBMC were harvested from the interface and

205 washed with PBS. Erythrocytes were removed with RBC Lysis Buffer (BioLegend).

206 The cell suspension was washed again, filtered through a $100 \mu \mathrm{m}$ cell strainer and

207 cryopreserved in freezing medium (HI FBS with $10 \%$ DMSO). Bronchoalveolar

208 lavage (BAL) was performed on the isolated right lung with $300 \mathrm{~mL}$ of PBS. Cells

209 were isolated by centrifugation of the BAL fluid (BALF) at $490 \times g$ for 5 min,

210 filtered through $100 \mu \mathrm{m}$ cell strainer, and cryopreserved in freeing medium. The

211 BALF supernatant was aliquoted, and frozen at $-80{ }^{\circ} \mathrm{C}$ for virus titration. For gene

212 expression analysis, pieces of cranial lung lobe tissues were submerged into

213 RNAlaterTM Stabilization Solution (Thermo Fisher Scientific), placed at room

214 temperature for $6 \mathrm{~h}$ and then stored at $-80{ }^{\circ} \mathrm{C}$ until use. Sampling of nasal secretions

215 was performed at 0, 1, 2, 3, 4 and 5 dpc using cotton swabs (one in each nostril,

216 Scientific Laboratory Supplies, Nottingham, UK). Swabs were then placed into virus

217 transport medium and processed as described previously (26).

\section{$218 \quad$ RNA extraction from fluids and tissues}

219 Total RNA was extracted from nasal swabs, BALF and serum using QIAamp Viral

220 RNA kit (QIAGEN, Manchester, UK) according to the manufacturer's instructions. 
221 Piece of lungs (200-300 mg) were weighed and homogenized using gentleMACS

222 Octo dissociator (Miltenyi Biotec) in RPMI 1640 medium in M tubes (Miltenyi

223 Biotec, Woking, UK). After centrifugation at $880 \times g$ for $5 \mathrm{~min}$, supernatants were

224 collected. Total RNA was extracted with RNAeasy kit (QIAGEN) according to the

225 manufacturer's instructions. Extracted RNA were stored at $-80{ }^{\circ} \mathrm{C}$ until use.

226 Virus titration by plaque assay

227 SwIAV titers in nasal swabs and BALF were determined by plaque assay as 228 previously described (22). MDCK cells were inoculated with 10-fold serially diluted 229 samples in MEM. After $1 \mathrm{~h}$ incubation at $37{ }^{\circ} \mathrm{C}$, cells were washed and incubated 230 for further $72 \mathrm{~h}$ at $37{ }^{\circ} \mathrm{C}$ in $5 \% \mathrm{CO}_{2}$ under an overlay medium consisting of MEM 231 with $0.21 \%$ BSA, 2 mM L-glutamine, $0.15 \%$ sodium bicarbonate, $10 \mathrm{mM}$ HEPES 232 (Merck), $1 \%$ penicillin/streptomycin, $0.01 \%$ Dextran DEAE (Merck), $0.6 \%$ agar 233 (Merck) and $2 \mu \mathrm{g} / \mathrm{mL}$ TPCK trypsin (Merck). The pfu were counted following 234 staining with $1 \%(\mathrm{w} / \mathrm{v})$ crystal violet (Merck).

\section{Viral RNA quantification by qRT-PCR}

236 PRRSV-2 RNA quantification in nasal swabs, BALF and serum was performed by 237 quantitative reverse-transcriptase PCR (qRT-PCR) using the one-step Quantinova ${ }^{\mathrm{TM}}$ 238 Probe RT-PCR kit (QIAGEN). Primers and a TaqMan probe were designed to 239 hybridize to a sequence in ORF7 (encoding N), and sequences are shown in Table 1.

240 To determine the viral RNA copies/mL in a sample, an RNA standard was 241 generated. PRRSV-2 RNA was used to amplify cDNA encoding the full-length of N 242 gene by RT-PCR (One-step RT-PCR, QIAGEN) using specific primers with the 243 forward containing a T7 promoter sequence at the 5' end. The PCR product was gel 244 purified (Illustra ${ }^{\text {TM }}$ GFX PCR DNA and Gel Band Purification Kit, Merck) and used 245 as template for in vitro transcription using the MEGAscript T7 Transcription Kit 246 (Thermo Fisher Scientific), according to the manufacturer's protocol. After DNase 247 treatment, the concentration of RNA was measured using a Nanodrop 248 spectrophotometer (Thermo Fisher Scientific), and the number of RNA 249 molecules $/ \mu \mathrm{L}$ was calculated using Avogadro's number $\left(6.023 \times 10^{23}\right)$. The qRT250 PCR was performed with $5 \mu \mathrm{L}$ of the eluted samples and $15 \mu \mathrm{L}$ of the master mix. 
251 Samples and standards were run in duplicate under the following conditions on a

252 Stratagene Mx3500P cycler (Agilent): reverse transcription at $45^{\circ} \mathrm{C}$ for $20 \mathrm{~min}$,

253 denaturation at $95{ }^{\circ} \mathrm{C}$ for $5 \mathrm{~min}, 35$ amplification cycles of denaturation at $95{ }^{\circ} \mathrm{C}$ for

$2545 \mathrm{~s}$ and combined annealing/extension at $60{ }^{\circ} \mathrm{C}$ for $30 \mathrm{~s}$. The viral genome copy

255 numbers were determined by interpolation of the standard curve and limit of

256 quantification was estimated at 60 copies $/ \mathrm{mL}$.

\section{Gene expression by RT-PCR}

258 Total RNA samples were treated with DNA-free Kit (Thermo Fisher Scientific).

259 Absence of residual DNA was verified by using RNA samples as a template for

260 SYBR Green PCR. Relative mRNA expression was evaluated by RT-PCR using the

261 QuantiNova SYBR Green RT-PCR Kit (QIAGEN). Primers of selected cytokines

262 and chemokines used in previous studies $(27,28)$ are listed in Table 1. RT-PCR

263 assays were validated and displayed an efficiency between $80 \%$ and $110 \%$.

264 Reactions utilized $5 \mu \mathrm{L}$ containing $100 \mathrm{ng}$ of RNA sample with $15 \mu \mathrm{L}$ of the master

265 mix. Samples were run in duplicate under the following conditions on a Stratagene

$266 \mathrm{Mx} 3500 \mathrm{P}$ cycler (Agilent): reverse transcription at $50{ }^{\circ} \mathrm{C}$ for $30 \mathrm{~min}$, denaturation at

$26795{ }^{\circ} \mathrm{C}$ for $5 \mathrm{~min}, 40$ amplification cycles of denaturation at $95{ }^{\circ} \mathrm{C}$ for $10 \mathrm{~s}$ and

268 appropriate combined annealing/extension temperature shown in Table 1 for $60 \mathrm{~s}$.

269 Melting curves were generated after each run to confirm the RT-PCR assay

270 specificity. Fold changes in gene expression were calculated using the delta-Cq

271 method with multiple housekeeping genes (29). The housekeeping genes RPS24

272 (ribosomal protein S24) and GAPDH (glyceraldehyde 3-phosphate dehydrogenase)

273 were found to be stably expressed in pig lung cells $(27,30)$ and were used as

274 reference genes to normalize the data. Samples from naïve group were used as

275 calibrators.

\section{Intracellular cytokine staining}

277 Cryopreserved PBMC and BAL cells were thawed, washed, and resuspended in

278 RPMI 1640 medium with $10 \%$ HI FBS and antibiotics. To assess the intracellular

279 cytokine production, $2 \times 10^{6}$ cells/well were seeded in a 96-well-round bottom tissue

280 culture plate with complete RPMI 1640 medium. After 5 h of resting at $37^{\circ} \mathrm{C}$, cells 
were re-stimulated with H3N2 CM5 (MOI 0.1) and PRRSV-2 16CB02 (MOI 0.1)

282 for $18 \mathrm{~h}$ at $37^{\circ} \mathrm{C}$. Cells cultured in complete RPMI 1640 medium only served as

283 negative controls. BD GolgiPlug at 1:1,000 (BD Biosciences, Wokingham, UK) was

284 added into the well for a further $4 \mathrm{~h}$ before staining. Cells stimulated with a cocktail

285 of PMA (phorbol 12-myristate 13-acetate) (20 ng; Merck), ionomycin (500 ng;

286 Merck) and BD GolgiPlug at 1:1,000 for $4 \mathrm{~h}$ was used as positive a control. Cells 287 were washed and stained with $50 \mu \mathrm{L}$ of surface marker antibodies listed 288 in Supplementary Table S2, and Near-IR Fixable LIVE/DEAD stain (Thermo 289 Fisher Scientific) diluted in PBS with $2 \% \mathrm{FBS}$ for $20 \mathrm{~min}$ at $4{ }^{\circ} \mathrm{C}$. After two 290 washing steps, the cells were fixed and permeabilized (BD Cytofix/Cytoperm kit;

291 BD Biosciences) for $30 \mathrm{~min}$ at $4{ }^{\circ} \mathrm{C}$. Intracellular cytokine staining (ICS) was 292 performed according to the manufacturer's directions using $50 \mu \mathrm{L}$ of cytokine mAbs 293 listed in Supplementary Table S2 diluted in Perm/Wash Buffer (BD Biosciences) 294 for $30 \mathrm{~min}$ at $4{ }^{\circ} \mathrm{C}$. Subsequently, cells were washed in Perm/Wash Buffer and fixed 295 with $2 \%$ PFA in PBS (Santa Cruz Biotechnology, Heidelberg, Germany). Cells 296 were analyzed with a BD LSRFortessa Flow Cytometer (BD Biosciences). Analysis 297 was performed with FlowJo version 10.6.2 (FlowJo, LLC). Compensation was set 298 according to single color staining controls. Isotype controls and fluorescence minus 299 one (FMO) controls were used to validate the staining and to set the gates.

300 Evaluation of anti-PRRSV antibody responses by ELISA and virus neutralization assay

302 To detect anti-PRRSV IgG, HI sera were tested using PrioCHECK PRRSV 303 Antibody ELISA Kit (Thermo Fisher Scientific) for detection of PRRSV N protein304 specific antibodies, according to the manufacturer's instructions. Optical density 305 (OD) was measured at $450 \mathrm{~nm}$ using a microplate reader (Synergy ${ }^{\mathrm{TM}}$ HT Multi306 Detection Microplate Reader, BioTek Instruments, USA). Percentage positivity (PP) 307 was calculated using following formula: $\mathrm{PP}=(\mathrm{OD}$ of sample $-\mathrm{OD}$ of negative 308 control) / (OD of positive control - OD of negative control) $\times 100$. The cut-off was 309 determined according to the supplier's protocol i.e., samples above 30 PP were

310 considered as positive. To measure anti-PRRSV IgG titers in serum, an ELISA using 311 PRRSV-2 infected cell lysate as antigen was used. Antigen was generated as 312 described elsewhere (31). Briefly, lysate was obtained by sonicating PRRSV-2 
313 16CB02 infected MARC-145 cell pellets in lysis buffer (1\% Triton X-100, $50 \mathrm{mM}$

314 borate, $150 \mathrm{mM} \mathrm{NaCl}, \mathrm{pH}$ 9). The supernatant was clarified and stored at $-80{ }^{\circ} \mathrm{C}$.

315 High-binding 96-well plates (Nunc Maxisorp ${ }^{\mathrm{TM}}$, Thermo Fisher Scientific) were

316 coated overnight at $4{ }^{\circ} \mathrm{C}$ with $100 \mu \mathrm{L}$ of lysate diluted at 1:40 in carbonate buffer

317 (Merck). After a blocking step with PBS with $4 \%$ milk for $1 \mathrm{~h}$, serial 2-fold

318 dilutions of HI serum were added in duplicates, starting from 1:40 and incubated for

$3191 \mathrm{~h}$ at room temperature (RT). Bound PRRSV-2-specific IgG was detected using

320 goat anti-pig IgG conjugated with horseradish peroxidase (HRP; Bio-Rad) diluted at

321 1:10,000 in blocking buffer for $1 \mathrm{~h}$ at RT. HRP enzymatic activity was revealed

322 using 3,3',5,5'-tetramethylbenzidine (TMB) substrate solution (Thermo Fisher

323 Scientific) for $5 \mathrm{~min}$ and was stopped by adding $1.2 \mathrm{M}$ sulfuric acid. Antibody

324 endpoint titers were determined as the highest dilution giving twice the OD of the

325 negative control wells (lysate coated well only). PRRSV neutralizing Ab titers were

326 assessed using an adapted protocol previously described (32). Briefly, serial 2-fold

327 dilutions of HI serum incubated with 400 TCID $_{50}$ of PRRSV-2 for $1 \mathrm{~h}$ at $37{ }^{\circ} \mathrm{C}$ were

328 added to MARC-145 cell monolayers. After 3 days incubation at $37{ }^{\circ} \mathrm{C}$, cells were

329 fixed and permeabilized (2 \% PFA $0.1 \%$ Triton X-100 in PBS) for $10 \mathrm{~min}$ at RT

330 and blocked with $10 \%$ goat serum in PBS. Cells were stained using an anti-PRRSV

$331 \mathrm{~N}$ mAb (SDOW17-A, Rural Technologies) diluted 1:1,600, followed by a secondary

332 goat anti-mouse IgG conjugated to HRP (Bio-Rad) diluted 1:1,000. PRRSV-2

333 positive cells were revealed using 3, 3'-diaminobenzidine substrate (DAB, Vector

334 Laboratories, Burlingame, USA) for $10 \mathrm{~min}$. Neutralizing Ab titers were calculated

335 as the reciprocal serum dilution that neutralized viral infection in $100 \%$ of the wells.

\section{Statistical analysis}

337 Data were analyzed with GraphPad Prism 8.0.1 software. As distribution was not

338 normal (Anderson-Darling test), the non-parametric unpaired Kruskal-Wallis test

339 followed by Dunn's correction was applied for multiple comparison (lung lesions

340 scores, viral load, proportion of immune cell subsets and genes expression). The

341 unpaired non-parametric Mann-Whitney test was used to compare data between 2

342 groups. The matched paired non-parametric Wilcoxon test was used to compare the

$343 \mathrm{~T}$ cell and antibody responses at day 0 and different timepoints within the same 344 group. 


\section{Results}

\section{PRRSV-2/H3N2 co-infection abrogates PRRS MLV-induced clinical protection}

347 To investigate whether concurrent infection with H3N2 and PRRSV-2 can interfere 348 with the efficacy of PRRS MLV vaccination, groups of 6 pigs were immunized with 349 a commercial PRRS MLV (Vac) or mock vaccinated with PBS (Ctrl) by 350 intramuscular injection (Figure 1A). Animals were challenged at 33 day post351 vaccination (dpv) with $5 \times 10^{6}$ pfu of a swIAV H3N2 field isolate (Ctrl $\left.+\mathrm{H} 3 \mathrm{~N} 2\right)$, $35210^{5}$ TCID $_{50}$ of a PRRSV-2 field isolate (Ctrl + PRRSV-2 and Vac + PRRSV-2) or 353 both viruses (Ctrl + PRRSV-2/H3N2 and Vac + PRRSV-2/H3N2) by intranasal 354 inoculation. Pigs were culled 5 days post-challenge $(38 \mathrm{dpv})$. A group of 6 pigs 355 remained untreated throughout the study and served as naïve controls. Clinical signs 356 and rectal temperature were monitored daily post-challenge (Supplementary 357 Tables S1 and S3). In the unvaccinated and H3N2 infected group (Ctrl $+\mathrm{H} 3 \mathrm{~N} 2)$, 358 1/6 pigs had an elevated temperature $\left(\geq 40{ }^{\circ} \mathrm{C}\right)$ after the challenge, $2 / 6$ pigs in the unvaccinated and PRRSV-2 infected group (Ctrl + PRRSV-2) and 1/6 pigs in the unvaccinated and co-infected group (Ctrl + PRRSV-2/H3N2) (Figure 1B). No other apparent clinical signs were observed after challenge (data not shown), which indicated that both field strain viruses induced a mild disease during this stage of infection, and that co-infection did not enhance clinical disease. None of the vaccinated pigs infected with PRRSV-2 (Vac + PRRSV-2) showed hyperthermia but 4/6 of the pigs vaccinated and co-infected with PRRSV-2/H3N2 (Vac + PRRSV2/H3N2) developed fever $\left(\geq 40^{\circ} \mathrm{C}\right)$ which lasted for 3 consecutive days in one pig

Following the experimental infection, microscopic examination of the lungs (Supplementary Table S3). collected at $5 \mathrm{dpc}$ revealed mild to moderate bronchointerstitial pneumonia in $\mathrm{H} 3 \mathrm{~N} 2$ $(\mathrm{Ctrl}+\mathrm{H} 3 \mathrm{~N} 2$ or $\mathrm{Ctrl}+\mathrm{PRRSV}-2 / \mathrm{H} 3 \mathrm{~N} 2)$ or PRRSV-2 infected groups (Ctrl + PRRSV-2 or Vac + PRRSV-2/H3N2) in comparison to the naïve controls or vaccinated group and infected with PRRSV-2 (Vac + PRRSV-2) (Supplementary

375 expansion and thickening of alveolar septa, and lymphocyte cuffing and histiocytic 376 cellular infiltration in the peribronchial and perivascular space as previously 
377 described after H3N2 $(33,34)$ and PRRSV $(23,35)$ infection. Immunohistochemistry

378 analysis indicated that IAV NP was observed in the relevant group, i.e., mainly

379 observed in cells within the epithelium of bronchi and bronchioles in $\mathrm{Ctrl}+\mathrm{H} 3 \mathrm{~N} 2$,

380 Ctrl + PRRSV-2/H3N2 and Vac + PRRSV-2/H3N2 groups, and not in naïve and

381 Ctrl + PRRSV-2 and Vac + PRRSV-2 groups (Supplementary Figures S2A).

382 Similarly, PRRSV $\mathrm{N}$ was localized, as expected, in alveolar and interstitial

383 macrophages in the Ctrl + PRRSV-2, Ctrl + PRRSV-2/H3N2, Vac + PRRSV-2 and

384 Vac + PRRSV-2/H3N2 groups (Supplementary Figures S2B).

The severity of lung lesions was compared between unvaccinated groups

387 after a single and co-infection using three different scoring systems, with similar

388 results (Figures 1C-E). Single infection of unvaccinated pigs with H3N2 or PRRSV-2 induced lung lesions (mean scores of 5.6 and 9.6, respectively) in comparison to naïve pigs (mean scores of 1), although significant differences in lung lesions were found only after PRRSV-2 infection ( $p<0.05$ vs naïve). Simultaneous PRRSV-2/H3N2 infection did not significantly increase the lung lesions in the unvaccinated group (mean score of 7) compared to single virus infection groups. To assess whether PRRS vaccine conferred protection, lung lesion scores were compared between Ctrl + PRRSV-2 and Vac + PRRSV-2 groups. A significant reduction of the score was observed in Vac + PRRSV-2 (mean score of 3.5) in comparison to Ctrl + PRRSV-2 group (mean score 9.6; $\mathrm{p}<0.05$ all scoring systems). However, PRRSV-2/H3N2 co-infection abrogated the protective effect of the PRRS MLV since the Vac + PRRSV-2/H3N2 animals exhibited high lung lesion scores in

400 comparison to Vac + PRRSV-2 animals (mean score 13.2 versus 3.5, respectively) $401 \quad(\mathrm{p}<0.05$, all scoring systems $)$.

Collectively, these results indicate that co-infection with $\mathrm{H} 3 \mathrm{~N} 2$ abrogates the PRRS MLV mediated protective effect, by enhancing lung lesions and clinical 404 disease.

407 To assess whether PRRSV-2/H3N2 co-infection affects the ability of PRRS MLV

408 vaccine to reduce virus loads, PRRSV-2 RNA was quantified in nasal swabs, BALF 
and serum (Figure 2). Shedding of PRRSV-2 was observed in nasal swabs from 3

410 to $5 \mathrm{dpc}$ in all PRRSV-2 challenged groups and not in the samples from naïve or Ctrl

$411+\mathrm{H} 3 \mathrm{~N} 2$ group (Figure 2A). At these early timepoints post-infection, we found great

412 variability within group. PRRSV-2 genome was detected in nasal swabs in 4/6 pigs

413 from Ctrl + PRRSV, in 1/6 pigs from Ctrl + PRRSV-2/H3N2, in 5/6 pigs from Vac

$414+$ PRRSV and in 1/6 pigs from Vac + PRRSV-2/H3N2. These data indicate that

415 PRRS MLV did not reduce viral shedding. Of note, PRRSV-2 RNA was detected in

416 only 1/6 in co-infected groups (Ctrl or Vac), suggesting that co-infection may reduce

417 PRRSV-2 viral shedding.

In the lower respiratory tract, high levels of PRRSV-2 RNA were measured 420 in BALF after single PRRSV-2 infection in both Ctrl + PRRSV-2 or Vac + PRRSV4212 groups and not in naïve and $\mathrm{Ctrl}+\mathrm{H} 3 \mathrm{~N} 2$ groups (Figure 2B). A reduction

422 (average 4-fold) of the PRRSV-2 RNA load was observed in the Vac + PRRSV-2 423 group compared to Ctrl + PRRSV-2 group although the difference was not 424 significant, indicating that intramuscularly administered PRRS MLV may help to 425 limit the viral replication in the lung at $5 \mathrm{dpc}$. Interestingly, in both vaccinated and 426 unvaccinated groups, there was a trend towards lower PRRSV-2 RNA loads in 427 BALF following the co-infection compared to the single infection. These results 428 indicate that co-infection seems to be efficient to reduce viral load (Figure 2B). In 429 the serum, PRRSV-2 RNA was also detected at $5 \mathrm{dpc}$ in Ctrl or Vac + PRRSV-2 430 groups and not in naïve and $\mathrm{Ctrl}+\mathrm{H} 3 \mathrm{~N} 2$ groups (Figure 2C). However, levels of 431 PRRSV-2 RNA were significantly reduced in Vac + PRRSV-2 compared to Ctrl + 432 PRRSV-2 (mean 4-fold, p<0.05) indicating that the vaccine conferred a degree of 433 protection. A significant reduction of PRRSV-2 viral RNA was also measured in 434 Ctrl + PRRSV-2/H3N2 group compared to Ctrl + PRRSV-2 (mean 19-fold; p<0.05). 435 The co-infection also slightly reduced PRRSV-2 RNA in Vac + PRRSV-2/swIAV 436 group as compared to the Vac + PRRSV-2 group, although the difference was not 437 statistically significant (mean 5-fold; $\mathrm{p}=0.06$ ).

H3N2 load was also assessed in nasal swabs and BALF. Virus titers were 439 detectable in nasal swabs of groups challenged with H3N2 (alone or with PRRSV-2) 440 and not in naïve and PRRSV-2 only infected animals (Figure 2D). After a single 
441 infection with $\mathrm{H} 3 \mathrm{~N} 2(\mathrm{Ctrl}+\mathrm{H} 3 \mathrm{~N} 2)$, a peak of the virus shedding was reached at 2

$442 \mathrm{dpc}$, followed by a plateau until 5 dpc. After PRRSV-2/H3N2 co-infection, the peak

443 of nasal shedding was also reached at $2 \mathrm{dpc}$ in the Ctrl + PRRSV-2/H3N2 group,

444 whereas this was seen earlier at $1 \mathrm{dpc}$ in Vac + PRRSV-2/H3N2 group. A significant

445 reduction of H3N2 was measured in the Ctrl + PRRSV-2/H3N2 at 5 dpc (mean 50-

446 fold; $\mathrm{p}<0.01$ ), however, this reduction disappeared in animals vaccinated with PRRS

447 MLV (Vac + PRRSV-2/H3N2 group) which exhibited similar pattern of H3N2

448 shedding to $\mathrm{Ctrl}+\mathrm{H} 3 \mathrm{~N} 2$.

In BALF, H3N2 was detected at low levels after the single $(\mathrm{Ctrl}+\mathrm{H} 3 \mathrm{~N} 2-$ 450 mean of $47 \mathrm{pfu} / \mathrm{mL})$ or after co-infection (Ctrl + PRRSV-2/H3N2 - mean of 2 $451 \mathrm{pfu} / \mathrm{mL}$ ) although these differences were not significant (Figure 2E). After co452 infection, PRRS MLV vaccination markedly increased the H3N2 titers in the Vac + 453 PRRSV-2/H3N2 group group compared to the non-immunized co-infected group 454 (mean 69 and $2 \mathrm{pfu} / \mathrm{mL}$ respectively, $\mathrm{p}=0.05$ ).

Together, these data indicated that PRRS MLV immunization reduced PRRSV-2 viraemia but did not reduce PRRSV-2 replication in the respiratory tract after challenge. PRRSV-2/H3N2 co-infection did not alter the effect of PRRS MLV

458 vaccination on PRRSV-2 load. However, in unimmunized animals, co-infection had 459 a beneficial effect by reducing PRRSV-2 viraemia and H3N2 shedding, with a trend 460 for lower PRRSV-2 and H3N2 loads in the BALF. However, the reduction of H3N2 461 viral load was reversed in PRRS MLV immunized animals.

\section{PRRSV-2-specific antibody responses}

464 We next determined whether PRRSV-2/H3N2 co-infection modulates the PRRS $465 \mathrm{MLV}$ induced $\mathrm{Ab}$ responses. $\mathrm{Ab}$ in serum were evaluated using a commercial 466 PRRSV N-specific ELISA at $0 \mathrm{dpv}, 20 \mathrm{dpv}$ and $5 \mathrm{dpc}$ (38 dpv) (Figure 3A). Sera 467 from immunized pigs at $20 \mathrm{dpv}$ were above the cut-off and displayed significant 468 levels of PRRSV N-specific Abs ( $\mathrm{p}<0.05$; 0 vs $20 \mathrm{dpv}$ ) indicating a seroconversion 469 following PRRS MLV immunization. There was no significant difference in the 470 level of PRRSV N-specific Abs between the Vac groups at $20 \mathrm{dpv}$. However, after 
471 the challenge, an increase of PRRSV N-specific Abs was detected in the Vac +

472 PRRSV-2/H3N2 group (p<0.05; 20 vs $38 \mathrm{dpv}$ ) but not in the Vac + PRRSV-2

473 group. The levels of PRRSV N-specific antibodies were comparable between Vac +

474 PRRSV-2 and Vac + PRRSV-2/H3N2 groups at $5 \mathrm{dpc}(\mathrm{p}=0.13)$.

Serum $\mathrm{Ab}$ titers were also measured by an in-house ELISA using lysate from PRRSV-2 16CB02 infected cells as antigens (Figure 3B). Consistent with the results found with the N-based ELISA test, significant anti-PRRSV-2 IgG titers

478 were measured after immunization ( $\mathrm{p}<0.05$; 0 vs $20 \mathrm{dpv}$ ) which were similar in both

$479 \mathrm{Vac}+$ PRRSV-2 and Vac + PRRSV-2/H3N2 groups ( $\mathrm{p}=0.13 ; 20 \mathrm{dpv})$. However, 480 after challenge, the Vac + PRRSV-2/H3N2 group had significantly higher Ab titers 481 compared to the Vac + PRRSV-2 group (mean Ab titer: 40,647 vs 17,126, 482 respectively; $\mathrm{p}<0.01$ ), suggesting that the co-infection with $\mathrm{H} 3 \mathrm{~N} 2$ enhanced the 483 recall of $\mathrm{B}$ cell responses primed by PRRS MLV immunization. Remarkably, none 484 of the sera collected at 20 and $38 \mathrm{dpv}$ were able to neutralize the PRRSV-2 16CB02 485 challenge strain (Figure 3C). The lack of neutralization might be explained by 486 differences between the vaccine and challenge strains (Supplementary Table S4).

These data indicated that PRRS MLV vaccination induced a significant 488 PRRSV-2-specific Ab response, which was enhanced after PRRSV-2/H3N2 co489 infection. In contrast, the PRRSV-2 only challenge did not significantly alter the magnitude of the specific antibody response (Figure 3C).

\section{PRRSV-2-specific T cell responses in PBMC}

493 In swine, conventional $\mathrm{CD}^{+} \mathrm{T}$ cells are defined as $\mathrm{CD}^{+} \mathrm{CD}^{+} \mathrm{CD}^{+} \alpha^{+/-} \mathrm{CD} 8 \beta^{-}$and $494 \mathrm{CD}^{+} \mathrm{T}$ cells as $\mathrm{CD}^{+} \mathrm{CD}^{-} \mathrm{CD}^{-} \beta^{+}(36,37)$. PRRSV-2 specific CD4 ${ }^{+}$and $\mathrm{CD}^{+} \mathrm{T}$ cell 495 responses were assessed by intracellular staining of IFN- $\gamma$, TNF, IL-2, IL-4, and IL49617 after in vitro restimulation of PBMC isolated at 0,20 and $38 \mathrm{dpv}$ (5 dpc) with 497 PRRSV-2 (Supplementary Figures S3 and S4). Overall, the frequencies of 498 cytokine-producing cells were low in $\mathrm{T}$ cell populations after PRRS MLV 499 immunization, as demonstrated previously (11) (Figure 4). Similar results were 500 obtained after the challenge with either PRRSV-2 alone or together with H3N2, 
501 although a substantial increase in IFN- $\gamma$ expressing $\mathrm{CD}^{+} \mathrm{T}$ cells was detected after

502 in the Vac + PRRSV-2/H3N2 group but not the Vac + PRRSV-2 group (mean 0.11

$503 \%$ and $0.06 \%$, respectively) (Figure 4B). Similarly, an increased proportion of

504 PRRSV-2-specific TNF expressing $\mathrm{CD}^{+} \mathrm{T}$ cell population was detected in these

505 two groups (mean $0.24 \%$ and $0.35 \%$ ), but this was not boosted after challenge. In

506 addition, no IL-2, IL-4 and IL-17 expressing CD4 ${ }^{+}$and IL-2 expressing CD ${ }^{+}$T

507 cells were detected after challenge (Figure 4 and supplementary Figure S5).

508 Overall, PRRS MLV immunization induced TNF and IFN- $\gamma$ expressing CD8 ${ }^{+}$

509 T cells in PBMC, which were not boosted by the subsequent PRRSV-2 or PRRSV-

510 2/H3N2 challenge. PRRSV-2-specific $\mathrm{CD}^{+} \mathrm{T}$ cell responses were weaker

511 compared to $\mathrm{CD}^{+} \mathrm{T}$ cells and there was no significance between the groups for any

512 of the measured cytokines.

\section{PRRSV-2- and H3N2- specific T cell responses in BALF}

515 Local $\mathrm{T}$ cell responses in the lung likely to play a role in controlling both IAV (38-

$51640)$ and PRRSV-2 infections and pathology $(11,41)$. We therefore assessed the $\alpha \beta$

517 and $\gamma \delta \mathrm{T}$ cell responses against both PRRSV-2 and H3N2 in BALF at 5 dpc. Cells

518 were restimulated with PRRSV-2 or H3N2, and cytokine production was assessed

519 by ICS. Staining of IFN- $\gamma$, TNF, IL-2 were performed for $\mathrm{CD}^{+}$and $\mathrm{CD}^{+} \alpha \beta \mathrm{T}$

520 cells, and staining of IFN- $\gamma$, TNF, IL-17 for $\gamma \delta$ T cells (Supplementary Figure S6),

521 which were divided into $\mathrm{CD}^{+}$and $\mathrm{CD} 2^{-}$subsets (42). $\mathrm{CD}^{+}$and $\mathrm{CD}^{+} \mathrm{T}$ cell

522 responses to PRRSV-2 overall were very low after single infection with PRRSV-2 in

523 either Ctrl or Vac groups (Figure 5A). However, T cell responses to PRRSV-2

524 stimulation were higher after PRRSV-2/H3N2 co-infection in both Ctrl and Vac

525 pigs. The highest proportion of $\mathrm{IL}^{-} 2^{+} \mathrm{CD}^{+}($mean $0.14 \%)$ and $\mathrm{IFN}-\gamma^{+} \mathrm{CD} 8^{+} \mathrm{T}$ cells

526 (mean $0.11 \%$ ) was observed in Ctrl + PRRSV-2/H3N2 group. In the Vac group,

527 PRRSV-2/H3N2 co-infection induced a proportion of IFN- $\gamma^{+} \mathrm{CD}^{+} \mathrm{T}$ cells (mean

$5280.23 \%$ ) and a significantly greater proportion of $\mathrm{TNF}^{+} \mathrm{CD}^{+} \mathrm{T}$ cells compared to

529 Vac + PRRSV-2 (mean $0.35 \%$; p<0.5). Similarly, co-infection induced the highest

530 proportion of IFN- $\gamma^{+} \mathrm{CD} 2^{+} \gamma \delta \mathrm{T}$ cells in the Vac group (mean $0.22 \%$ ), and IL-17 
$531 \mathrm{CD} 2^{+} \gamma \delta \mathrm{T}$ cells in the $\mathrm{Ctrl}$ group (mean $0.30 \%$ ) (Figure 5B). Higher frequencies of

$532 \mathrm{TNF}^{+}$and $\mathrm{IL}_{-1} 7^{+} \mathrm{CD} 2^{-} \gamma \delta \mathrm{T}$ cells were detected in the Ctrl + PRRSV-2/H3N2

533 compared to Ctrl + PRRSV-2 group (mean $0.73 \%$ versus $0.31 \%$ and mean $0.40 \%$

534 versus $0.23 \%$, respectively) though this was not statistically significant. In

535 vaccinated pigs, co-infection increased the frequency of $\mathrm{TNF}^{+}$(mean $0.47 \%$ ) and

$536 \mathrm{IL}-17^{+}$(mean $0.51 \%$ ) $\mathrm{CD}^{-} \gamma \delta \mathrm{T}$ cells, although these differences did not reach

537 statistical significance.

538 Stimulation of BALC with H3N2 also showed the greatest frequencies of 539 cytokine producing $\mathrm{T}$ cells in co-infected animals (Figure 6A). The proportion of

540 IL-2 expressing $\mathrm{CD}^{+} \mathrm{T}$ cells in the Ctrl + PRRSV-2/H3N2 group was higher

541 compared to $\mathrm{Ctrl}+\mathrm{H} 3 \mathrm{~N} 2$ (mean $0.23 \%$ versus $0.05 \%$ ). Similarly, the frequency of

$542 \mathrm{IFN}-\gamma^{+} \mathrm{CD}^{+} \mathrm{T}$ cells in the $\mathrm{Ctrl}+\mathrm{PRRSV}-2 / \mathrm{H} 3 \mathrm{~N} 2$ group was higher compared to

543 the $\mathrm{Ctrl}+\mathrm{H} 3 \mathrm{~N} 2$ (mean $0.35 \%$ versus $0.07 \%$ ), but none of these differences reached

544 statistical significance. A greater frequency of $\mathrm{TNF}^{+} \mathrm{CD} 2^{+} \gamma \delta \mathrm{T}$ cells was again

545 measured in the Ctrl + PRRSV-2/H3N2 group compared to Ctrl + H3N2 group

546 (mean $0.29 \%$ versus $0.01 \%$ ) (Figure 6B). Similar results were obtained for $\mathrm{TNF}^{+}$

547 and $\mathrm{IL}-17^{+} \mathrm{CD} 2^{-} \gamma \delta \mathrm{T}$ cells in the Ctrl + PRRSV-2/H3N2 group compared to the Ctrl

$548+\mathrm{H} 3 \mathrm{~N} 2$ group (mean $1.18 \%$ versus $0.23 \%$ and $1.02 \%$ versus $0.28 \%$,

549 respectively). Intriguingly, lower $\mathrm{H} 3 \mathrm{~N} 2$-specific cytokine producing $\mathrm{T}$ cell

550 responses were induced by the co-infection in PRRS MLV vaccinated group in

551 comparison to the unvaccinated group although no statistically significant

552 differences were observed (Figures 6A and 6B).

553 Overall, these data indicate that PRRSV-2/H3N2 co-infection induces the 554 highest frequency of cytokine producing T cells in response to both PRRSV-2 and $555 \mathrm{H} 3 \mathrm{~N} 2$ in BAL, although these did not reach statistical significance, most likely due 556 to the sample size. The data also highlight the effect of PRRS MLV vaccination on 557 the immune responses after the co-infection. The increased PRRSV-specific, but 558 lowered H3N2-specific T cell responses in the Vac + PRRSV-2/H3N2 compared to 559 the Ctrl + PRRSV-2/H3N2 suggest that PRRS MLV may potentially drive the host 560 immune response toward PRRSV-specific, and rather away from the H3N2-specific 561 responses. 


\section{Cytokine expression in the lung}

563 To further characterize the immune responses in the lungs of singly and co-

564 infected pigs, the gene expression of a panel of cytokines and chemokines was

565 assessed in lung tissues and data were normalized to the naïve group (Figure 7).

566 After single infection with $\mathrm{H} 3 \mathrm{~N} 2$, an elevated mRNA expression of pro-

567 inflammatory cytokines TNF and IFN- $\gamma$ (mean fold change of 2.22 and 1.86,

568 respectively), and anti-inflammatory cytokines IL-10 and TGF- $\beta$ (mean fold change

569 of 1.92 and 2.40 respectively), was observed (Figure 7). In PRRSV-2-infected

570 group (Ctrl + PRRSV-2), a modest increase of TNF, IL-12p40 and IL-10 transcripts

571 (mean fold change of 1.67, 1.69 and 1.80, respectively) was measured. After co-

572 infection (Ctrl + PRRSV-2/H3N2), TNF, IFN- $\gamma$, IL-12p40, IL-4 and CXCL-13

573 transcripts were all up-regulated (mean fold change of 2.43, 1.86, 3.47, 2.49 and

574 2.83, respectively). In both vaccinated single and co-infected groups, an up-

575 regulation of TNF (mean fold change 2.0 in both Vac groups) and IFN- $\gamma$ transcripts

576 were quantified (mean fold change 1.8 in both Vac groups). Notably both IL-10

577 (mean fold change of 2.5 and 2.4, respectively) and TGF- $\beta$ (mean fold change of 1.6

578 and 2.0, respectively) mRNA expression was also upregulated in these vaccinated

579 groups. A significant down-regulation of IL-12p40 mRNA was observed in Vac +

580 PRRSV-2/H3N2 pigs compared to the Ctrl + PRRSV-2/H3N2 group ( $\mathrm{p}<0.05)$.

581 Despite a lack of statistical significance, these data suggest that upregulation

582 of inhibitory cytokines gene expression in the lungs of previously immunized pigs

583 might have influenced responses to H3N2 and PRRSV-2. 
Discussion

587 The PRDC is responsible for major economic losses in the pig industry worldwide.

588 The PRDC commonly results from mixed infections, in combination with 589 environmental stressors (16). PRRSV and swIAV, alone or in combination, are two 590 major viral pathogens involved in the PRDC, often leading to secondary infections

591 with opportunistic bacteria. Previous experimental in vivo PRRSV/swIAV co-

592 infection studies reported variable outcomes in terms of clinical or virological

593 parameters, which may reflect differences in the timing of infections $(18,43,44)$.

594 Superinfection, i.e., infection of pigs with H1N1 3 days later a primary infection

595 with PRRSV-1 led to more severe disease and a delayed shedding of H1N1

596 compared to pigs that were infected 14 days later (45), whereas simultaneous

597 PRRSV-1/H1N1 co-infection did not alter the clinical and virological course of

598 infection of either virus (44). Several studies have also assessed the effect of

599 concurrent infections and superinfections on vaccines efficacy and demonstrated

600 that PRRSV infection decreases the efficacy of swIAV (46), Mycoplasma

601 hyopneumoniae (47), and classical swine fever (48) vaccines although underlying

602 mechanisms involved have not been demonstrated. Whilst vaccination with PRRS

603 MLV is widely practiced in efforts to control PRRS, swIAV vaccination is less often

604 used. Reflecting the field situation, a recent study experimentally assessed the 605 impact of swIAV superinfection on vaccination with PRRS MLV efficacy and found

606 a delay in MLV replication and Ab responses, but this did not affect vaccine 607 efficacy (19). In our study here, we investigated the effect of PRRSV-2/H3N2 co608 infection on the protection provided by a commercial PRRS MLV vaccine. The 609 PRRS MLV protected against clinical disease, reduced lung pathology and viremia, 610 but not virus shedding, as previously reported (14,49-51). However, PRRSV611 2/H3N2 co-infection abrogated the protective effect of PRRS MLV vaccination on 612 clinical disease and pathology. Co-infection did not affect the vaccine-induced 613 reduction in PRRSV-2 load and enhanced $\mathrm{CD}^{+} \mathrm{T}$ cell responses in the lung and $\mathrm{Ab}$ 614 responses. In contrast, co-infection in non-immunized animals had a beneficial 615 effect by reducing PRRSV-2 viremia and H3N2 virus loads in BALF and nasal 616 swabs at day 5 post-infection and did not affect clinical signs or pathology. 
618 We sought to dissect the mechanisms underlying these opposing effects of co619 infection in immunized and non-immunized animals at day 5 post-infection. The 620 reduced PRRSV load in unimmunized co-infected animals has been suggested to be 621 due to the early type I IFN response triggered by H3N2, and interference and/or 622 competition for substrates required for replication (19,52). In this study, analysis of 623 gene expression in the lungs, did not reveal significant differences in the expression 624 of IFN- $\alpha$, which may be due to the timing of sampling (5 days post-infection). 625 However, there was significantly more IL-12p40 gene expression, in the lung, and a 626 trend for higher number of $\mathrm{H} 3 \mathrm{~N} 2$-specific $\mathrm{T}$ cell responses (IL-2 ${ }^{+} \mathrm{CD} 4^{+}, \mathrm{IFN}-\gamma^{+}$ $627 \mathrm{CD}^{+}, \mathrm{IL}_{-1} 7^{+} \mathrm{CD} 2^{-} \gamma \delta$ and $\mathrm{TNF}^{+} \mathrm{CD} 2^{-} \gamma \delta \mathrm{T}$ cells) and PRRSV-2-specific $\mathrm{T}$ cell 628 responses (IL-17 ${ }^{+} \mathrm{CD}^{-} \gamma \delta \mathrm{T}$ cells $\mathrm{TNF}^{+} \mathrm{CD}^{-} \gamma \delta \mathrm{T}$ cells) in the BALF of the co629 infected unimmunized animals. This trend for enhanced cell-mediated response, 630 may have contributed to the control of virus replication in the lung in the co-infected 631 groups compared to the singly infected groups. In vaccinated pigs, co-infection 632 induced a greater PRRSV-specific $\mathrm{TNF}^{+} \mathrm{CD}^{+} \mathrm{T}$ cell response in the BALF and an 633 increased $\mathrm{Ab}$ response. However, this was not the case for the specific response to $634 \mathrm{H} 3 \mathrm{~N} 2$, and the cytokine gene expression profile in the lung did not differ 635 significantly from the singly infected animals. The precise mechanism for 636 abrogation of the PRRS MLV protective effect against lung injury by co-infection 637 remains unclear but might be due to exuberant cytokine production by other cell 638 types producing more pro-inflammatory cytokines such as TNF and IL-6 (53), 639 which were not detected at the timepoint sampled here. Although effectors CD8 ${ }^{+} \mathrm{T}$ 640 cells in the BAL aids to eliminate PRRSV-infected cells, the local response might 641 contribute to pulmonary inflammation and injury. Alternatively, co-infection may 642 have enhanced the production of low affinity non-neutralizing antibodies that may 643 augment infection and exacerbate disease.

Whilst PRRS MLV vaccines can provide a significant clinical benefit, the 645 protection against virus shedding is limited which may drive the evolution of 646 PRRSV (54,55). This has been attributed to these vaccines being weakly 647 immunogenic, especially for cellular responses (8). In line with previous reports, we 648 confirmed that PRRS MLV vaccinated pigs recorded lower lung lesions, viraemia 649 and viral load in lungs after a single infection with PRRSV-2, which was associated 
650 with a robust, although non-neutralizing, antibody response and a weak peripheral

651 PRRSV-specific T cell response $(11,14,50,51)$.

652 PRRSV has been shown to suppress host immune response through the 653 induction of regulatory $\mathrm{T}$ cells (56), alteration of peripheral NK cell cytotoxic 654 activity (57), and inhibition of cytokine responses $(58,59)$. Moreover, PRRS MLV 655 vaccine can induce systemic secretion of IL-10 (60). It is therefore plausible that the 656 vaccine used in our study may exhibit similar immunomodulatory features. We 657 observed that, in comparison to unvaccinated pigs, lungs from vaccinated pigs co658 infected with PRRSV-2/H3N2 displayed a lower level of pro-inflammatory IL$65912 \mathrm{p} 40$ gene expression, along with a trend for higher expression of anti660 inflammatory IL-10 and TGF- $\beta$ as measured by RT-qPCR. In a study on 661 Litomosoides sigmodontis helminth infection (60), helminth-infected mice exhibit 662 lower HA-specific antibody responses post-IAV vaccination, linked with a higher 663 viral load in the lung compared to non-infected mice. The level of HA-specific 664 induced by the vaccine was restored after the blockade of IL-10 using an anti-IL-10 665 receptor mAb. PRRS MLV vaccination may have potentially impaired the host 666 immune response to $\mathrm{H} 3 \mathrm{~N} 2$ and perhaps facilitated its replication. In addition to the 667 highest lung pathology seen in the PRRS MLV vaccinated co-infected pigs, the 668 beneficial effect of the co-infection in reducing H3N2 viral loads was also abrogated 669 in vaccinated pigs. These data suggests that PRRS MLV initiates a strong PRRS 670 specific response and may suppress H3N2 specific responses leading to increased 671 pathology and poor disease outcome.

Whilst confirmatory studies are required, the demonstration that co-infection 674 with H3N2 can abrogate the clinical protective effect of PRRSV MLV suggests that 675 better control measures against swIAV might be warranted to further increase 676 protection against PRRSV. Large number of herds are endemically infected with 677 swIAV and suffer intermittent bouts of disease. SwIAV contributes to suboptimal 678 weight gain and reproductive performance and is occasionally associated with fever679 induced abortion in sows (61). Immunization can be a cost-effective control measure 680 to combat swIAV, but the rapid evolution of the virus is a major obstacle (62). Not 
681 all swIAV-endemic countries use vaccines to control the disease, for example,

682 current UK policy does not involve immunization against swIAV, although it is used

683 in some European countries and widely in the USA (63). We have used recent field

684 PRRS and swIAV strains from Thailand. As in the UK, immunization of pigs

685 against swIAV is not mandatory in Thailand, although some farms use the

686 GRIPORK $^{\circledR}$ vaccine (Hipra, Spain) containing inactivated H1N1 and H3N2 strains.

687 Inactivated vaccines induce neutralizing $\mathrm{Ab}$ against the immunizing strain but do not

688 induce sufficient heterologous protection due to the rapid viral escape that occurs

689 through antigenic drift of the surface glycoproteins (64), so that coincident infection

690 with PRRS and swIAV remains possible. There is a need to develop more broadly

691 protective influenza vaccines that could provide a better control of swIAV, reducing

692 the zoonotic risk (65), the contribution to the PRDC and its potentially harmful

693 effect on PRRS vaccine efficacy.

694

695 Data availability

696 The raw data supporting the conclusions of this article will be made available by the 697 authors, without undue reservation.

699 Competing interests

700 The authors declare that the research was conducted in the absence of any 701 commercial or financial relationships that could be construed as a potential conflict 702 of interest.

\section{$704 \quad$ Funding}

705 This study was supported by the Newton Fund UK-China-Philippines-Thailand 706 Swine and Poultry Research Initiative award BB/R01275X/1 (Broadly protective 707 vaccines for porcine reproductive and respiratory syndrome and swine influenza 708 virus infections), UKRI Biotechnology and Biological Sciences Research Council 
709 (BBSRC) Institute Strategic Programme and Core Capability Grants to The Pirbright

710 Institute (BBS/E/I/00007031 and BBS/E/I/00007037 and BBS/E/I/00007039).

\section{Acknowledgements}

713 The authors would like to acknowledge The Pirbright Institute Flow Cytometry

714 Unit, and Animal Services Team for animal care and their invaluable help for

715 providing samples. We would also like to thank Miriam Pedrera and Rebecca K.

716 McLean for their assistance during post-mortems.

717

\section{Author Contributions}

719 ET, SG, NW and SK acquired funding for the project. ET, SG and TC contributed to

720 the conception, design, and coordination of the study. TC and EM performed

721 experiments. TC acquired, analyzed, and interpreted the data. ET, SG, EM, EV,

722 VM, BP, AM, TM, and ME contributed to sampling during post-mortem. FS carried

723 out pathological analysis. TC wrote the first draft of the manuscript. ET, SG, NW,

724 and SK edited and revised the manuscript. All authors approved the submitted

725 version.

726

727 
728

729 1. Holtkamp DJ, Kliebenstein JB, Neumann EJ, Zimmerman JJ, Rotto HF,

730

731

732

733

734

735

736

737

738

739

740

741

742

743

744

745

746

747

748

749

750

751

752

753

754

755

756

757

758

759

760

761

762

763

764

765

766

767

\section{References} Yoder TK, et al. Assessment of the economic impact of porcine reproductive and respiratory syndrome virus on United States pork producers. J Swine Heal Prod. (2013) 21:72-84.

2. Stadejek T, Larsen LE, Podgórska K, Bøtner A, Botti S, Dolka I, et al. Pathogenicity of three genetically diverse strains of PRRSV Type 1 in specific pathogen free pigs. Vet Microbiol. (2017) 209:13-19. doi:10.1016/j.vetmic.2017.05.011

3. Weesendorp E, Rebel JMJ, Popma-De Graaf DJ, Fijten HP, StockhofeZurwieden N. Lung pathogenicity of European genotype 3 strain porcine reproductive and respiratory syndrome virus (PRRSV) differs from that of subtype 1 strains. Vet Microbiol. (2014) 174:127-38. doi:10.1016/j.vetmic.2014.09.010

4. Montaner-Tarbes S, del Portillo HA, Montoya M, Fraile L. Key Gaps in the Knowledge of the Porcine Respiratory Reproductive Syndrome Virus (PRRSV). Front Vet Sci. (2019) 6:38. doi:10.3389/fvets.2019.00038

5. Gebhardt JT, Tokach MD, Dritz SS, DeRouchey JM, Woodworth JC, Goodband RD, et al. Postweaning mortality in commercial swine production. I: Review of non-infectious contributing factors. Transl Anim Sci. (2020) 4: 485-506. doi:10.1093/tas/txaa052

6. Pejsak Z, Stadejek T, Markowska-Daniel I. Clinical signs and economic losses caused by porcine reproductive and respiratory syndrome virus in a large breeding farm. Vet Microbiol. (1997) 55:317-22. doi:10.1016/s03781135(96)01326-0

7. Walker PJ, Siddell SG, Lefkowitz EJ, Mushegian AR, Adriaenssens EM, Alfenas-Zerbini $\mathrm{P}$, et al. Changes to virus taxonomy and to the International Code of Virus Classification and Nomenclature ratified by the International Committee on Taxonomy of Viruses (2021). Arch Virol. 2021 (2021) 166: 2633-48. doi:10.1007/s00705-021-05156-1

8. Zhou L, Ge X, Yang H. Porcine reproductive and respiratory syndrome modified live virus vaccine: A "leaky" vaccine with debatable efficacy and safety. Vaccines. (2021) 9:362. doi:10.3390/vaccines9040362

9. Nan Y, Wu C, Gu G, Sun W, Zhang Y-J, Zhou E-M. Improved Vaccine against PRRSV: Current Progress and Future Perspective. Front Microbiol. (2017) 8:1635. doi:10.3389/fmicb.2017.01635

10. Geldhof MF, Vanhee M, Van Breedam W, Van Doorsselaere J, Karniychuk UU, et al. Comparison of the efficacy of autogenous inactivated Porcine Reproductive and Respiratory Syndrome Virus (PRRSV) vaccines with that of commercial vaccines against homologous and heterologous challenges. 
11. Kick AR, Amaral AF, Cortes LM, Fogle JE, Crisci E, Almond GW, et al. The T-Cell Response to Type 2 Porcine Reproductive and Respiratory Syndrome Virus (PRRSV). Viruses. (2019) 11:796. doi:10.3390/v11090796

12. Osorio FA, Galeota JA, Nelson E, Brodersen B, Doster A, Wills R, et al. Passive transfer of virus-specific antibodies confers protection against reproductive failure induced by a virulent strain of porcine reproductive and respiratory syndrome virus and establishes sterilizing immunity. Virology. (2002) 302:9-20. doi:10.1006/viro.2002.1612

13. Lopez OJ, Oliveira MF, Alvarez Garcia E, Kwon BJ, Doster A, Osorio FA. Protection against Porcine Reproductive and Respiratory Syndrome Virus (PRRSV) infection through passive transfer of PRRSV-neutralizing antibodies is dose dependent. Clin Vaccine Immunol. (2007) 14:269-75. doi:10.1128/CVI.00304-06

14. Kick AR, Amaral AF, Frias-De-Diego A, Cortes LM, Fogle JE, Crisci E, et al. The Local and Systemic Humoral Immune Response Against Homologous and Heterologous Strains of the Type 2 Porcine Reproductive and Respiratory Syndrome Virus. Front Immunol. (2021) 12:637613. doi:10.3389/fimmu.2021.637613

15. Rahe MC, Murtaugh MP. Mechanisms of adaptive immunity to porcine reproductive and respiratory syndrome virus. Viruses. (2017) 9:148. doi:10.3390/v9060148

16. Saade G, Deblanc C, Bougon J, Marois-Créhan C, Fablet C, Auray G, et al. Coinfections and their molecular consequences in the porcine respiratory tract. Vet Res. (2020) 51:80. doi:10.1186/s13567-020-00807-8

17. Choi YK, Goyal SM, Joo HS. Retrospective analysis of etiologic agents associated with respiratory diseases in pigs. Can Vet J. (2003) 44:735-37.

18. Van Reeth K, Nauwynck H, Pensaert M. Dual infections of feeder pigs with porcine reproductive and respiratory syndrome virus followed by porcine respiratory coronavirus or swine influenza virus: A clinical and virological study. Vet Microbiol. (1996) 48:325-35. doi:10.1016/0378-1135(95)00145-x

19. Renson P, Deblanc C, Bougon J, Le Dimna M, Gorin S, Mahé S, et al.

803 20. Hoffmann E, Stech J, Guan Y, Webster RG, Perez DR. Universal primer set 
807

808

809

810

811

812

813

814

815

816

817

818

819

820

821

822

823

824

825

826

827

828

829

830

831

832

833

834

835

836

837

838

839

840

841

842

843

844

845

846 847

Acute Influenza A virus outbreak in an enzootic infected sow herd: Impact on viral dynamics, genetic and antigenic variability and effect of maternally derived antibodies and vaccination. PLoS One. (2019) 14:e0224854. doi:10.1371/journal.pone.0224854

22. Holzer B, Morgan SB, Martini V, Sharma R, Clark B, Chiu C, et al. Immunogenicity and Protective Efficacy of Seasonal Human Live Attenuated Cold-Adapted Influenza Virus Vaccine in Pigs. Front Immunol. (2019) 10:2625. doi:10.3389/fimmu.2019.02625

23. Morgan SB, Frossard JP, Pallares FJ, Gough J, Stadejek T, Graham SP, et al. Pathology and Virus Distribution in the Lung and Lymphoid Tissues of Pigs Experimentally Inoculated with Three Distinct Type 1 PRRS Virus Isolates of Varying Pathogenicity. Transbound Emerg Dis. (2016) 63:285-95. doi:10.1111/tbed.12272

24. Morgan SB, Hemmink JD, Porter E, Harley R, Shelton H, Aramouni M, et al. Aerosol Delivery of a Candidate Universal Influenza Vaccine Reduces Viral Load in Pigs Challenged with Pandemic H1N1 Virus. J Immunol. (2016) 196:5014-23. doi:10.4049/jimmunol.1502632

25. Gauger PC, Vincent AL, Loving CL, Henningson JN, Lager KM, Janke BH, et al. Kinetics of Lung Lesion Development and Pro-Inflammatory Cytokine Response in Pigs With Vaccine-Associated Enhanced Respiratory Disease Induced by Challenge With Pandemic (2009) A/H1N1 Influenza Virus. Vet Pathol. (2012) 49:900-12. doi:10.1177/0300985812439724

26. McNee A, Smith T, Holzer B, Clark B, Bessell E, Guibinga G, et al. Establishment of a Pig Influenza Challenge Model for Evaluation of Monoclonal Antibody Delivery Platforms. J Immunol. (2020) 205:648-60. doi:10.4049/jimmunol.2000429

27. Bordet E, Blanc F, Tiret M, Crisci E, Bouguyon E, Renson P, et al. Porcine reproductive and respiratory syndrome virus type 1.3 Lena triggers conventional dendritic cells 1 activation and thelper 1 immune response without infecting dendritic cells. Front Immunol. (2018) 9:2299. doi:10.3389/fimmu.2018.02299

28. Liu G, Wang Y, Jiang S, Sui M, Wang C, Kang L, et al. Suppression of lymphocyte apoptosis in spleen by CXCL13 after porcine circovirus type 2 infection and regulatory mechanism of CXCL13 expression in pigs. Vet Res. (2019) 50:17. doi:10.1186/s13567-019-0634-2

29. Vandesompele J, De Preter K, Pattyn F, Poppe B, Van Roy N, De Paepe A, et al. Accurate normalization of real-time quantitative RT-PCR data by geometric averaging of multiple internal control genes. Genome Biol. (2002) 3:research0034. doi:10.1186/gb-2002-3-7-research0034

30. Maisonnasse P, Bouguyon E, Piton G, Ezquerra A, Urien C, Deloizy C, et al. The respiratory DC/macrophage network at steady-state and upon influenza 
848

infection in the swine biomedical model. Mucosal Immunol. (2016) 9:835-49. doi:10.1038/mi.2015.105

31. Chrun T, Lacôte S, Urien C, Richard CA, Tenbusch M, Aubrey N, et al. A DNA vaccine encoding the Gn ectodomain of Rift valley fever virus protects mice via a humoral response decreased by DEC205 targeting. Front Immunol. (2019) 10:860. doi:10.3389/fimmu.2019.00860

32. Robinson SR, Li J, Nelson EA, Murtaugh MP. Broadly neutralizing antibodies against the rapidly evolving porcine reproductive and respiratory syndrome virus. Virus Res. (2015) 203:56-65. doi:10.1016/j.virusres.2015.03.016

33. Martini V, Paudyal B, Chrun T, McNee A, Edmans M, Atangana Maze E, et al. Simultaneous Aerosol and Intramuscular Immunization with Influenza Vaccine Induces Powerful Protective Local T Cell and Systemic Antibody Immune Responses in Pigs. J Immunol. (2021) 206:652-63. 10.4049/jimmunol.2001086

34. Lyoo KS, Kim JK, Jung K, Kang BK, Song D. Comparative pathology of pigs infected with Korean H1N1, H1N2, or H3N2 swine influenza A viruses. Virol J. (2014) 11:170. doi:10.1186/1743-422X-11-170

35. Halbur PG, Paul PS, Frey ML, Landgraf J, Eernisse K, Meng XJ, et al. Comparison of the pathogenicity of two US porcine reproductive and respiratory syndrome virus isolates with that of the Lelystad virus. Vet Pathol. (1995) 32:648-60. doi:10.1177/ 030098589503200606

36. Edmans M, McNee A, Porter E, Vatzia E, Paudyal B, Martini V, et al. Magnitude and Kinetics of $\mathrm{T}$ Cell and Antibody Responses During H1N1pdm09 Infection in Inbred Babraham Pigs and Outbred Pigs. Front Immunol. (2021) 11:604913. doi:10.3389/fimmu.2020.604913

37. Talker SC, Koinig HC, Stadler M, Graage R, Klingler E, Ladinig A, et al. Magnitude and kinetics of multifunctional $\mathrm{CD} 4+$ and $\mathrm{CD} 8 \beta+\mathrm{T}$ cells in pigs infected with swine influenza A virus. Vet Res. (2015) 46:52. doi:10.1186/s13567-015-0182-3

38. Brown DM, Lee S, Garcia-Hernandez M de la L, Swain SL. Multifunctional CD4 Cells Expressing Gamma Interferon and Perforin Mediate Protection against Lethal Influenza Virus Infection. J Virol. (2012) 86:6792-803. doi:10.1128/JVI.07172-11

39. McMaster SR, Wilson JJ, Wang H, Kohlmeier JE. Airway-Resident Memory CD8 T Cells Provide Antigen-Specific Protection against Respiratory Virus Challenge through Rapid IFN- $\gamma$ Production. J Immunol (2015) 195:203-9. doi:10.4049/jimmunol.1402975

40. Palomino-Segura M, Latino I, Farsakoglu Y, Gonzalez SF. Early production of IL-17A by $\gamma \delta \mathrm{T}$ cells in the trachea promotes viral clearance during 
888

889

890

891

892

893

894

895

896

897

898

899

900

901

902

903

904

905

906

907

908

909

910

911

912

913

914

915

916

917

918

919

920

921

922

923

924

925

926

927

928

influenza infection in mice. Eur $J$ Immunol. (2020) 50:97-109. doi:10.1002/eji.201948157

41. Nazki S, Khatun A, Jeong CG, Mattoo SUS, Gu S, Lee SI, et al. Evaluation of local and systemic immune responses in pigs experimentally challenged with porcine reproductive and respiratory syndrome virus. Vet Res. (2020) 51:66. doi:10.1186/s13567-020-00789-7

42. Sedlak C, Patzl M, Saalmüller A, Gerner W. CD2 and CD8 $\alpha$ define porcine $\gamma \delta \mathrm{T}$ cells with distinct cytokine production profiles. Dev Comp Immunol. (2014) 45:97-106. doi:10.1016/j.dci.2014.02.008

43. Pol JM, van Leengoed LA, Stockhofe N, Kok G, Wensvoort G. Dual infections of PRRSV/influenza or PRRSV/Actinobacillus pleuropneumoniae in the respiratory tract. Vet Microbiol. (1997) 55:259-64. doi:10.1016/s03781135(96)01323-5

44. Pomorska-Mól M, Podgórska K, Czyżewska-Dors E, Turlewicz-Podbielska H, Gogulski M, Włodarek J, Łukomska A. Kinetics of single and dual simultaneous infection of pigs with swine influenza A virus and porcine reproductive and respiratory syndrome virus. J Vet Intern Med. (2020) 34(5):1903-13. doi:10.1111/jvim.15832

45. Van Reeth K, Nauwynck H, Pensaert M. Clinical effects of experimental dual infections with porcine reproductive and respiratory syndrome virus followed by swine influenza virus in conventional and colostrum-deprived pigs. $J$ Vet Med B Infect Dis Vet Public Health. (2001) 48:283-92. doi:10.1046/j.14390450.2001.00438.x

46. Kitikoon P, Vincent AL, Jones KR, Nilubol D, Yu S, Janke BH, et al. Vaccine efficacy and immune response to swine influenza virus challenge in pigs infected with porcine reproductive and respiratory syndrome virus at the time of SIV vaccination. Vet Microbiol. (2009) 139:235-44. doi:10.1016/j.vetmic.2009.06.003

47. Thacker EL, Thacker BJ, Young TF, Halbur PG. Effect of vaccination on the potentiation of porcine reproductive and respiratory syndrome virus (PRRSV)-induced pneumonia by Mycoplasma hyopneumoniae. Vaccine. (2000) 18:1244-52. doi:10.1016/s0264-410x(99)00395-3

48. Suradhat S, Kesdangsakonwut S, Sada W, Buranapraditkun S, Wongsawang $S$, Thanawongnuwech $R$. Negative impact of porcine reproductive and respiratory syndrome virus infection on the efficacy of classical swine fever vaccine. Vaccine. (2006) 24:2634-42. doi:10.1016/j.vaccine.2005.12.010

49. Oh T, Park KH, Yang S, Jeong J, Kang I, Park C, et al. Evaluation of the efficacy of a trivalent vaccine mixture against a triple challenge with Mycoplasma hyopneumoniae, PCV2, and PRRSV and the efficacy comparison of the respective monovalent vaccines against a single challenge. BMC Vet Res. (2019) 15:342. doi:10.1186/s12917-019-2091-6 
929

930

931

932

933

934

935

936

937

938

939

940

941

942

943

944

945

946

947

948

949

950

951

952

953

954

955

956

957

958

959

960

961

962

963

964

965

966

967

968

969

50. Jeong J, Kim S, Park C, Park KH, Kang I, Park SJ, et al. Commercial porcine reproductive and respiratory syndrome virus (PRRSV)-2 modified live virus vaccine against heterologous single and dual Korean PRRSV-1 and PRRSV-2 challenge. Vet Rec. (2018) 182:485. doi:10.1136/vr.104397

51. Sattler T, Pikalo J, Wodak E, Revilla-Fernández S, Steinrigl A, Bagó Z, et al. Efficacy of live attenuated porcine reproductive and respiratory syndrome virus 2 strains to protect pigs from challenge with a heterologous Vietnamese PRRSV 2 field strain. BMC Vet Res. (2018) 14:133. doi:10.1186/s12917-0181451-y

52. Fleming DS, Miller LC, Tian Y, Li Y, Ma W, Sang Y. Impact of porcine arterivirus, influenza $\mathrm{B}$, and their coinfection on antiviral response in the porcine lung. Pathogens. (2020) 9:934. doi:10.3390/pathogens9110934

53. Munoz FM, Cramer JP, Dekker CL, Dudley MZ, Graham BS, Gurwith M, et al. Vaccine-associated enhanced disease: Case definition and guidelines for data collection, analysis, and presentation of immunization safety data. Vaccine. (2021) 39:3053-66. doi:10.1016/j.vaccine.2021.01.055

54. Costers S, Vanhee M, Van Breedam W, Van Doorsselaere J, Geldhof M, Nauwynck HJ. GP4-specific neutralizing antibodies might be a driving force in PRRSV evolution. Virus Res. (2010) 154:104-13. doi: 10.1016/j.virusres.2010.08.026

55. Zhang Z, Zhou L, Ge X, Guo X, Han J, Yang H. Evolutionary analysis of six isolates of porcine reproductive and respiratory syndrome virus from a single pig farm: MLV-evolved and recombinant viruses. Infect Genet Evol. (2018) 66:111-19. doi: 10.1016/j.meegid.2018.09.024

56. Nedumpun T, Sirisereewan C, Thanmuan C, Techapongtada P, Puntarotairung R, Naraprasertkul S, et al. Induction of porcine reproductive and respiratory syndrome virus (PRRSV)-specific regulatory T lymphocytes (Treg) in the lungs and tracheobronchial lymph nodes of PRRSV-infected pigs. Vet Microbiol. (2018) 216:13-19. doi:10.1016/j.vetmic.2018.01.014

57. Renukaradhya GJ, Alekseev K, Jung K, Fang Y, Saif LJ. Porcine reproductive and respiratory syndrome virus-Induced immunosuppression exacerbates the inflammatory response to porcine respiratory coronavirus in pigs. Viral Immunol. (2010) 23:457-66. doi:10.1089/vim.2010.0051

58. Subramaniam S, Kwon B, Beura LK, Kuszynski CA, Pattnaik AK, Osorio FA. Porcine reproductive and respiratory syndrome virus non-structural protein 1 suppresses tumor necrosis factor-alpha promoter activation by inhibiting NF-kB and Sp1. Virology. (2010) 406:270-9. doi:10.1016/j.virol.2010.07.016

59. Ke H, Han M, Zhang Q, Rowland R, Kerrigan M, Yoo D. Type I interferon suppression-negative and host mRNA nuclear retention-negative mutation in nsp $1 \beta$ confers attenuation of porcine reproductive and respiratory syndrome 
$970 \quad$ virus in pigs. Virology. (2018) 517:177-87. doi:10.1016/j.virol.2018.01.016

971 60. Hartmann W, Brunn ML, Stetter N, Gagliani N, Muscate F, Stanelle-Bertram

972 S, et al. Helminth Infections Suppress the Efficacy of Vaccination against

973 Seasonal Influenza. Cell Rep. (2019) 29:2243-2256.e4.

974 doi:10.1016/j.celrep.2019.10.051

975 61. Janke B. H. (2013). Clinicopathological features of Swine influenza. Curr

976 Top Microbiol Immunol. 370:69-83. doi:10.1007/82_2013_308

977 62. Rajao DS, Anderson TK, Kitikoon P, Stratton J, Lewis NS, Vincent AL.

978 Antigenic and genetic evolution of contemporary swine H1 influenza viruses

979 in the United States. Virology. (2018) 518:45-54.

980 doi:10.1016/j.virol.2018.02.006

981 63. Mancera Gracia JC, Pearce DS, Masic A, Balasch M. Influenza A Virus in 982 Swine: Epidemiology, Challenges and Vaccination Strategies. Front Vet Sci. $983 \quad$ (2020) 7:647. doi:10.3389/fvets.2020.00647

984 64. Huang KY, Rijal P, Schimanski L, Powell TJ, Lin TY, McCauley JW, et al. 985 Focused antibody response to influenza linked to antigenic drift. J Clin Invest. 986 (2015) 125:2631-45. doi:10.1172/JCI81104

987 65. Lorbach JN, Nelson SW, Lauterbach SE, Nolting JM, Kenah E, McBride DS, 988 et al. Influenza Vaccination of Swine Reduces Public Health Risk at the 
991 Table 1: Primers used for RT-qPCR and RT-PCR

\begin{tabular}{|c|c|c|c|c|c|c|c|c|}
\hline Primers & Sequences $\left(5^{\prime}-3^{\prime}\right)$ & $\mu \mathbf{M}$ & $\operatorname{Tm}\left({ }^{\circ} \mathrm{C}\right)$ & Size (bp) & Eff (\%) & $\mathbf{R}^{2}$ & Slope & PMID \\
\hline \multirow{3}{*}{ N PRRSV-2 } & F: GCAAAATCAGTCCAGAGGCAAG & 400 & 60 & 183 & 98.6 & 0.99 & -3.33 & - \\
\hline & R: TGACAGGGTGCAAGTTCCAG & 400 & & & & & & - \\
\hline & P: FAM-CCCATTTCCCTCTTGCGACCGAAGATGACG-BHQ & 250 & & & & & & - \\
\hline \multirow[t]{2}{*}{ IFN- $\alpha$} & F: TCTGCAAGGTTCCCAATGG & 350 & 60 & 69 & 100 & 0.99 & -3.32 & 30333837 \\
\hline & R: GGCATTGCAGCTGAGTAGCA & 350 & & & & & & \\
\hline \multirow{2}{*}{ IFN- $\gamma$} & F: TGGTAGCTCTGGGAAACTGAATG & 350 & 60 & 79 & 89.3 & 0.89 & -3.60 & 30333837 \\
\hline & R: TGGCTTTGCGCTGGATCT & 350 & & & & & & \\
\hline \multirow{2}{*}{ TNF } & $F: T G G T G G T G C C G A C A G A T G$ & 350 & 60 & 64 & 108 & 0.96 & -3.12 & 30333837 \\
\hline & R:CAGCCTTGGCCCCTGAA & 350 & & & & & & \\
\hline \multirow[t]{2}{*}{ IL-4 } & F: GCCGGGCCTCGACTGT & 350 & 63 & 68 & 94.2 & 0.99 & -3.46 & 30333837 \\
\hline & R:TCCGCTCAGGAGGCTCTTC & 350 & & & & & & \\
\hline \multirow[t]{2}{*}{ IL-6 } & F: CTGCTTCTGGTGATGGCTACTG & 350 & 60 & 69 & 101.1 & 0.99 & -3.29 & 30333837 \\
\hline & R:GGCATCACCTTTGGCATCTT & 350 & & & & & & \\
\hline \multirow[t]{2}{*}{ IL-12p40 } & F: GGAGCACCCCACATTCCTACT & 350 & 60 & 68 & 92.7 & 0.98 & -3.51 & 30333837 \\
\hline & R:TTCTCTTTTGTTCTTGCCCTGAA & 350 & & & & & & \\
\hline \multirow[t]{2}{*}{ IL-21 } & F: AAATAGTCATCTGCCTGATGGTCAT & 350 & 60 & 76 & 103 & 0.97 & -3.25 & 30333837 \\
\hline & R:AGGCGATCTTGTCCTTGGAA & 350 & & & & & & \\
\hline \multirow[t]{2}{*}{ CXCL-13 } & F: ATCTCTGCTTCTCGTGCTG & 350 & 60 & 180 & 92 & 0.99 & -3.52 & 30819249 \\
\hline & R:ACTTCTCTGGTTGGACATCC & 350 & & & & & & \\
\hline \multirow[t]{2}{*}{ IL-10 } & F: GAGCCAACTGCAGCTTCCA & 350 & 60 & 65 & 109.8 & 0.98 & -3.10 & 30333837 \\
\hline & R:TCAGGACAAATAGCCCACTAGCTT & 350 & & & & & & \\
\hline \multirow[t]{2}{*}{ TGF- $\beta$} & F: GAAGCGCATCGAGGCCATTC & 350 & 60 & 162 & 91.1 & 1 & -3.55 & 30333837 \\
\hline & R:GGCTCCGGTTCGACACTTTC & 350 & & & & & & \\
\hline \multirow[t]{2}{*}{ RPS24 } & F: AAGGAACGCAAGAACAGAATGAA & 350 & 60 & 62 & 94.2 & 0.99 & -3.46 & 30333837 \\
\hline & R:TTTGCCAGCACCAACGTTG & 350 & & & & & & \\
\hline \multirow[t]{2}{*}{ GAPDH } & F: CACCATCTTCCAGGAGCGA & 350 & 60 & 51 & 101.5 & 0.98 & -3.28 & 30333837 \\
\hline & R:CCAGCATCACCCCACTTGAT & 350 & & & & & & \\
\hline
\end{tabular}

F: Forward; R: Reverse; P: Probe 


\section{Figures}

A

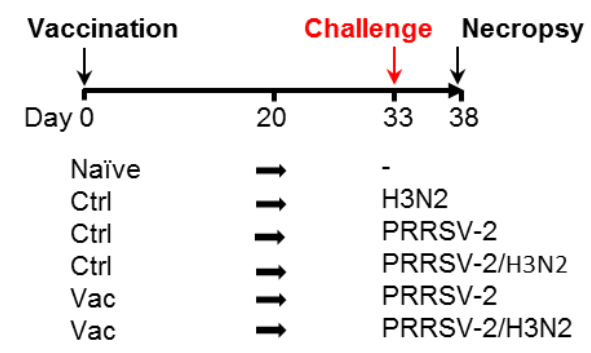

B

\begin{tabular}{cc}
\hline Group & $\begin{array}{c}\text { Number of pigs with body } \\
\text { temperature } \geq 40^{\circ} \mathrm{C} \text { after } \\
\text { the challenge }\end{array}$ \\
\hline Naive & $0 / 6$ \\
Ctrl + H3N2 & $1 / 6$ \\
Ctrl + PRRSV-2 & $2 / 6$ \\
Ctrl + PRRSV-2/H3N2 & $1 / 6$ \\
Vac + PRRSV-2 & $0 / 6$ \\
Vac + PRRSV-2/H3N2 & $4 / 6$ \\
\hline
\end{tabular}

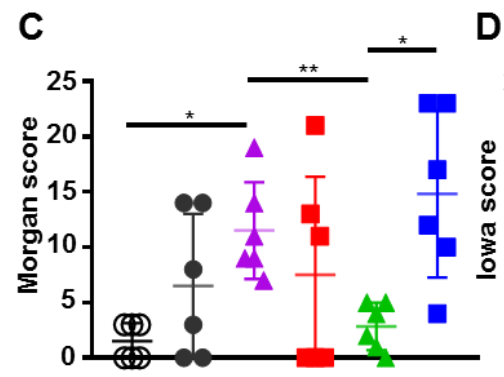

D $\quad$ E

O Naïve Ctrl + H3N2 $\Delta$ Ctrl + PRRSV-2 $\square$ Ctrl + PRRSV-2/H3N2 $\Delta$ Vac + PRRSV-2

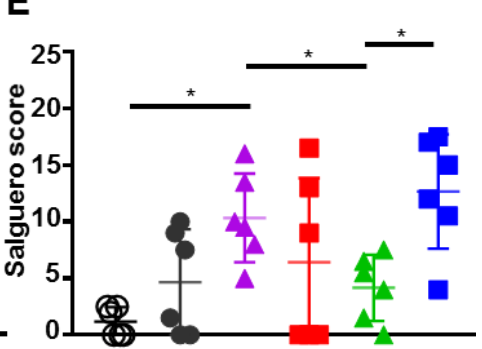

Vac + PRRSV-2/H3N2

995

996 Figure 1: Clinical signs and lung lesions. (A) Pigs were vaccinated 997 intramuscularly with Ingelvac PRRS ${ }^{\circledR}$ MLV (Vac) or with PBS (Ctrl) or were 998 untreated (naïve group). Thirty-three days after the vaccination, pigs were 999 challenged by intranasal inoculation with H3N2, PRRSV-2 or simultaneously with

1000 PRRSV-2/H3N2. Nasal swabs were daily taken after the challenge and pigs were 1001 culled 5 days later (38 days post-vaccination (dpv)). Sera and PBMC were collected 1002 at 0, 20 and $38 \mathrm{dpv}$. Clinical signs and rectal temperature were monitored daily after 1003 the challenge (dpc). (B) Table indicating the number of pigs that developed fever 1004 after the challenge. (C-E) Lungs sections were scored for histopathological lesions 1005 (C; Morgan score), lesions with presence of influenza NP-positive cells (D; Iowa 1006 score) or lesions with presence of PRRSV N-positive cells (E; Salguero score). Each 1007 symbol represents an individual animal within the indicated group ( $n=6$ per group).

1008 The horizontal lines represent mean \pm SD. Comparisons between 2 group were 1009 analyzed using Mann-Whitney test and asterisks indicate significant differences (*p $1010<0.05 ; * * p<0.01)$. 


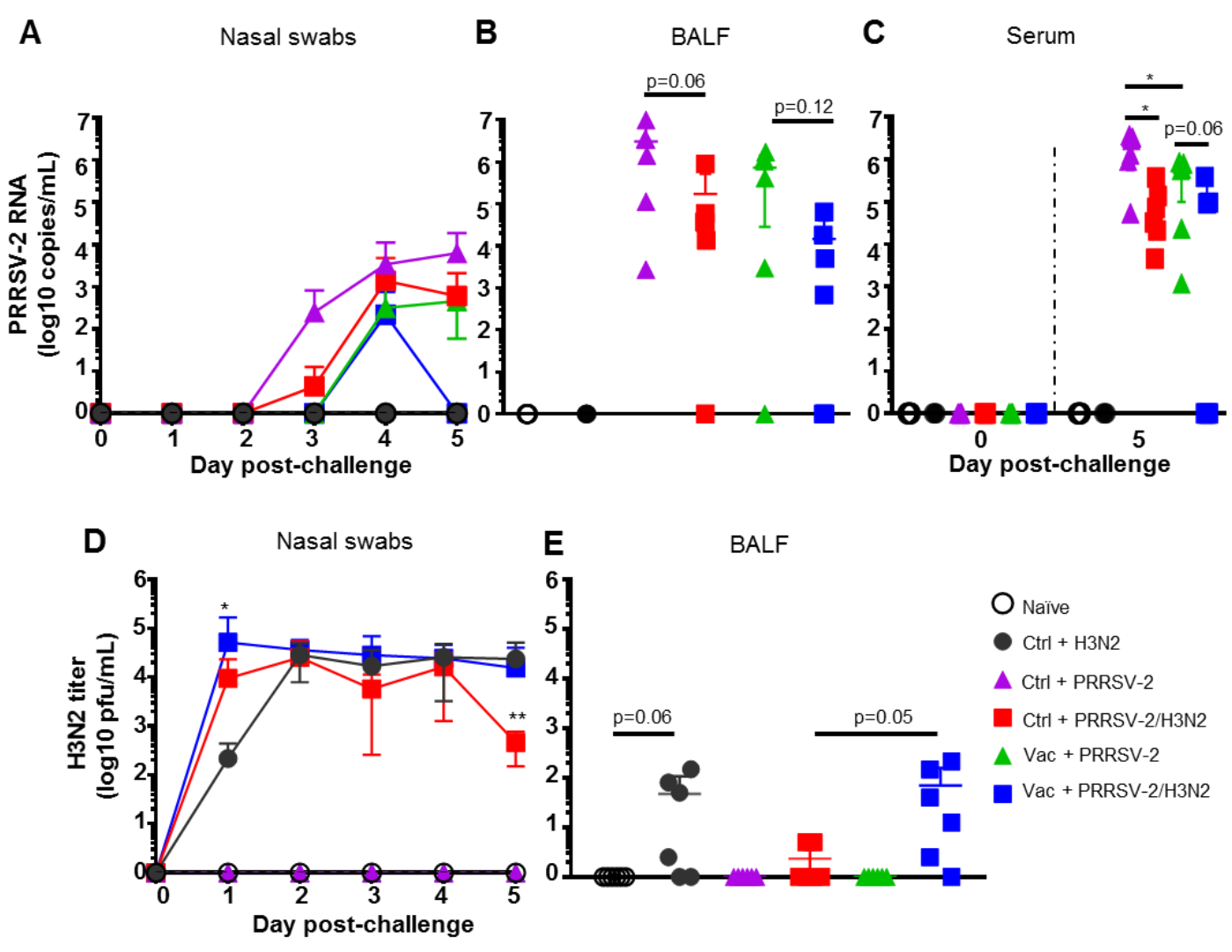

1011

1012 Figure 2: PRRSV-2 and H3N2 loads. Quantification of PRRSV-2 viral RNA in the

1013 nasal swabs (A), BALF (B) and serum (C) were determined by qRT-PCR. H3N2

1014 titers in nasal swabs (D) and BALF (E) were determined by plaque assay. The mean

1015 values (A and D) or individual values (B, C, D and $\mathbf{E}$ ) for each group \pm SD are

1016 indicated ( $\mathrm{n}=6$ per group). $\mathrm{P}$ values were determined using Mann-Whitney test and 1017 asterisks indicate significant differences $(* p<0.05)$. 
A

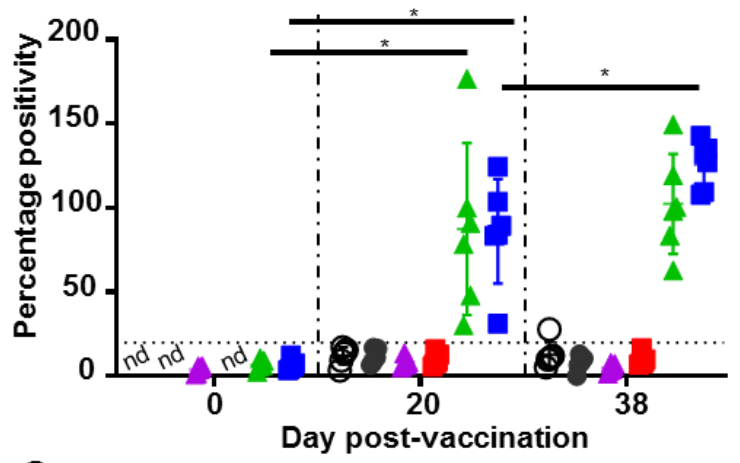

C

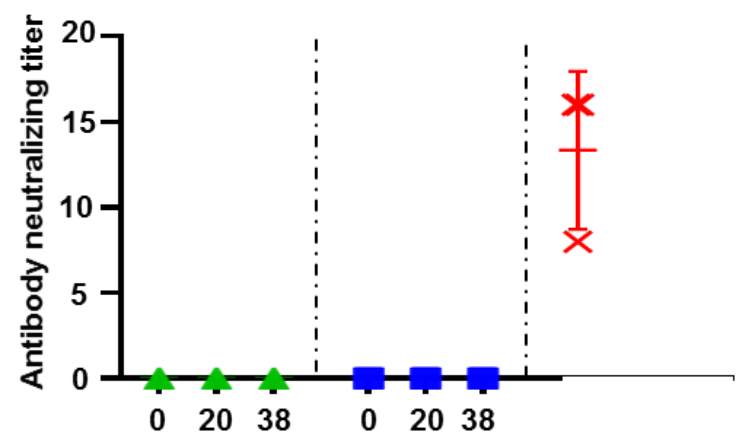

Day post-vaccination
B

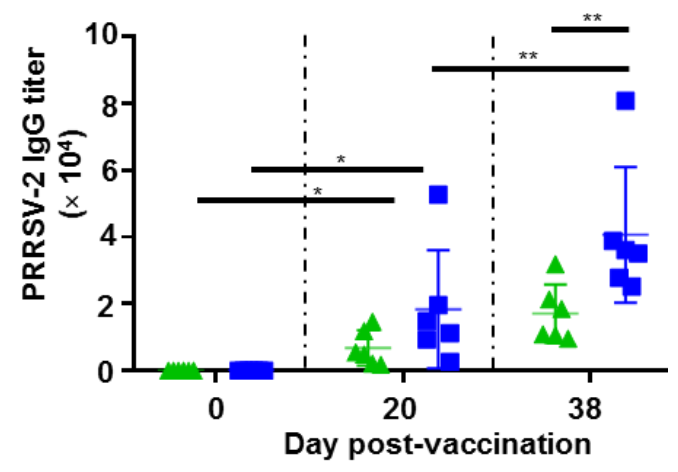

Naïve

- $\mathrm{Ctrl}+\mathrm{H} 3 \mathrm{~N} 2$

A Ctrl + PRRSV-2

C $\mathrm{Crl}+\mathrm{H} 3 \mathrm{~N} 2$

$\Delta$ Vac + PRRSV-2

- Vac + PRRSV-2/H3N2

$\times$ Positive control

1020 Figure 3: Antibody responses against PRRSV-2. (A) Detection of PRRSV N-

1021 specific Abs in the serum at 0,20 and $38 \mathrm{dpv}$ was performed with a commercial

1022 ELISA test. The positive threshold is indicated with dashed lines. (B) PRRSV-

1023 specific Ab titers were measured in the sera of PRRS-immunized pigs at 0, 20 and

$102438 \mathrm{dpv}$ with an-in house ELISA test. (C) Levels of virus neutralizing Ab titer in the

1025 serum of PRRS-vaccinated pig at 0, 20 and 38 are shown. Sera from PRRSV-2

1026 infected pigs from an unrelated study were used as positive controls (red symbols).

1027 Each pig serum is shown as a symbol within the indicated group ( $\mathrm{n}=6$ per group)

1028 and the mean $\pm \mathrm{SD}$ is represented. The comparison between the percentage

1029 positivity values or $\mathrm{Ab}$ titers measured at 20 and $38 \mathrm{dpv}$ versus at $0 \mathrm{dpv}$ for each

1030 vaccinated group were performed using the Wilcoxon test. Comparisons between

1031 groups were made using the Mann-Whitney test. Asterisks indicate significant

1032 differences $(* \mathrm{p}<0.05 ; * \mathrm{p}<0.01)$. nd: not determined. 
A
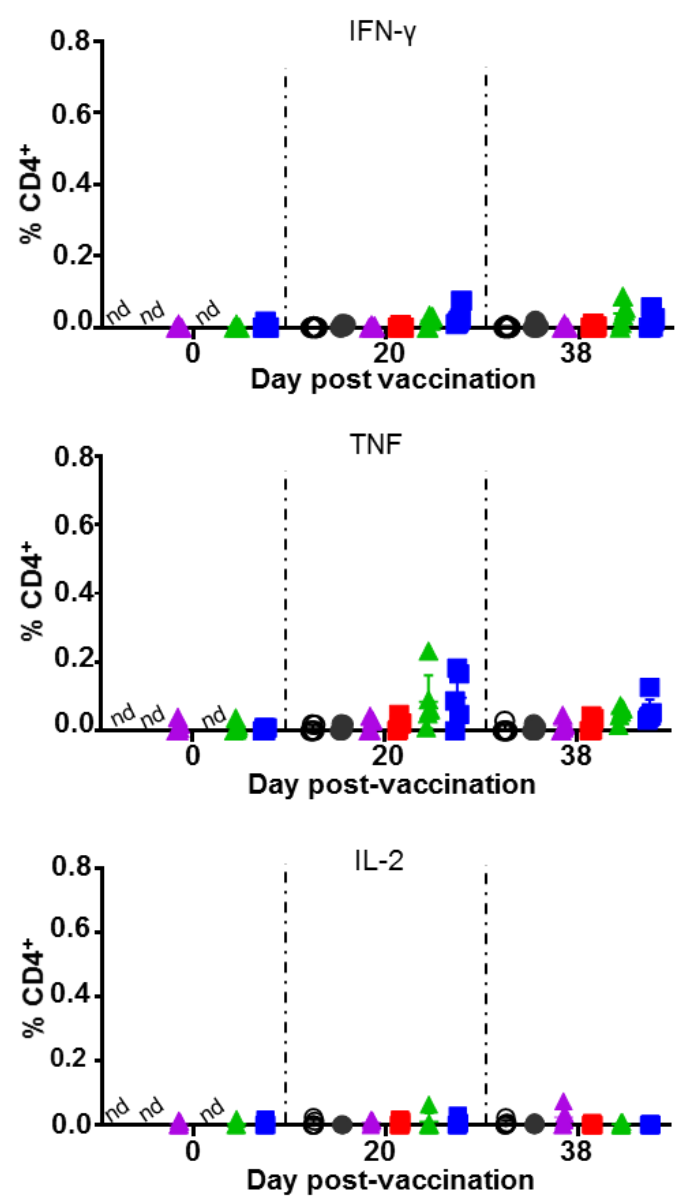

B
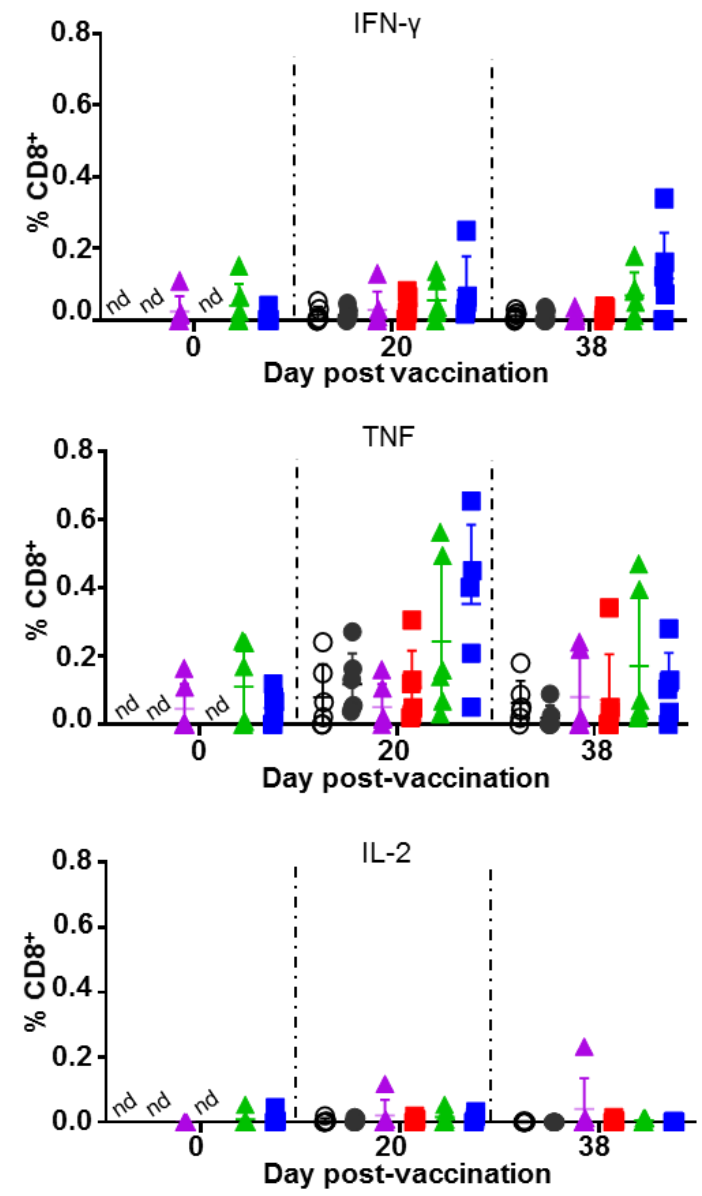

1036 Figure 4: PRRSV-2- specific T cell responses in PBMC. PBMC isolated at 0, 20 and $38 \mathrm{dpv}$ were restimulated in vitro for 18h with PRRSV-2 (MOI 0.1) or cultured

1038 with medium. Intracellular staining of IFN- $\gamma$, TNF, IL-2, was performed and 1039 frequencies of IFN- $\gamma, \mathrm{TNF}$ and IL-2 producing $\mathrm{CD}^{+}(\mathbf{A})$ and $\mathrm{CD}^{+} \beta^{+}(\mathbf{B}) \mathrm{T}$ cells 1040 were analyzed. The corrected frequencies values are shown (percentage of cytokine1041 producing cells subtracted with medium only). Data for individual pigs and the 1042 group mean \pm SD are displayed (n=5-6 per group). The Wilcoxon test was used to 1043 compare the $\mathrm{T}$ cell responses at day 0 and $20 \mathrm{dpv}$ within the same group. 1044 Comparisons between 2 groups were performed using Mann-Whitney test. nd: not 1045 determined. 


\section{A $\quad \alpha \beta T$ cells - PRRSV-2}

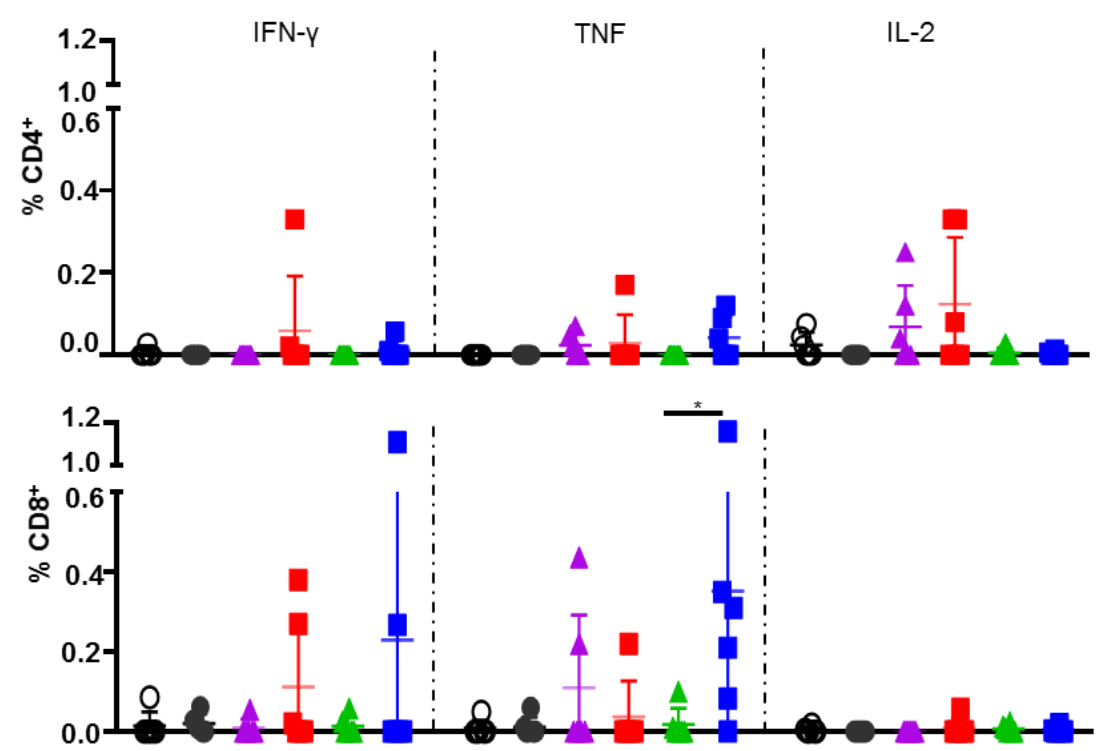

B Y YT cells-PRRSV-2
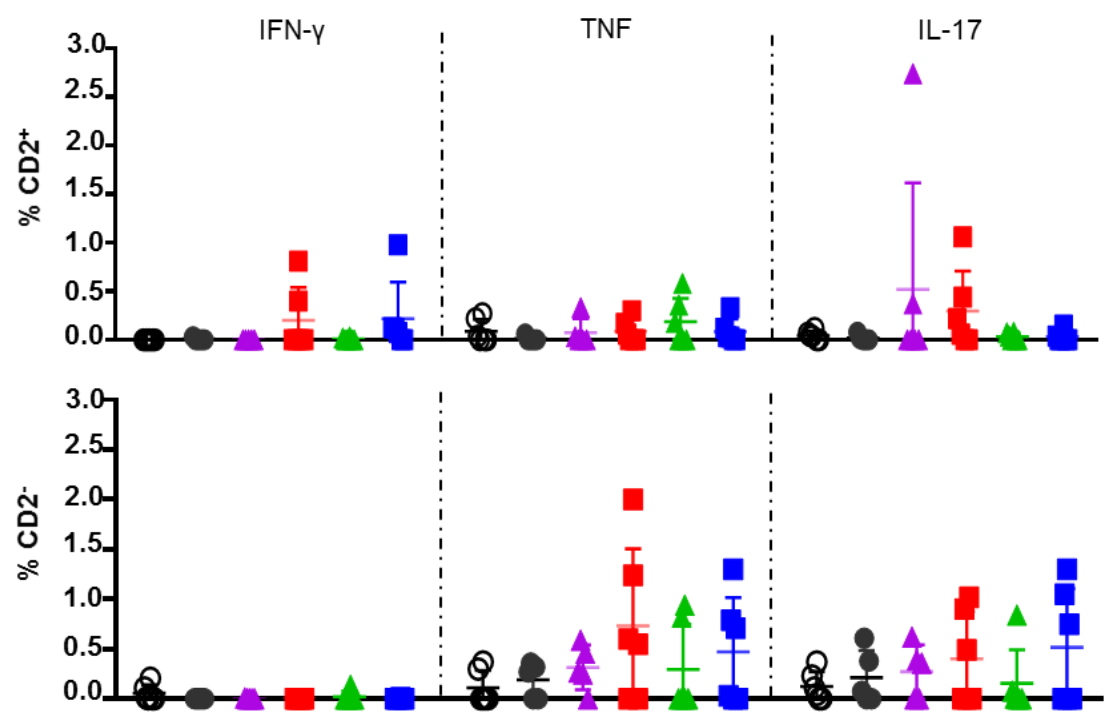

O Naïve Ctrl + H3N2 $\Delta$ Ctrl + PRRSV-2 $\square$ Ctrl + PRRSV-2/H3N2 $\Delta$ Vac + PRRSV-2 $\square$ Vac + PRRSV-2/H3N2

1048 Figure 5: PRRSV-2- specific T cell responses in bronchoalveolar lavage. Cells

1049 isolated from BAL at $5 \mathrm{dpc}$ were restimulated with PRRSV-2 (MOI 0.1) or cultured

1050 with medium. (A) Frequency of IFN- $\gamma-$, TNF- and IL-2-producing CD4 ${ }^{+}$and CD8 $\beta^{+}$

1051 T cells are shown. (B) Frequency of IFN- $\gamma-$, TNF- and IL-17-producing CD2 ${ }^{+}$and

$1052 \mathrm{CD}^{-} \gamma \delta \mathrm{T}$ cells are represented. The corrected frequencies (percentage of cytokine-

1053 producing cells subtracted with medium only) of each individual pig and the group

1054 mean \pm SD are displayed ( $n=5-6$ per group). Comparisons between groups were made using Mann-Whitney test. Asterisk indicates significant difference (*p < 0.05$)$. 


\section{A $\quad \alpha \beta T$ cells $-\mathrm{H} 3 \mathrm{~N} 2$}

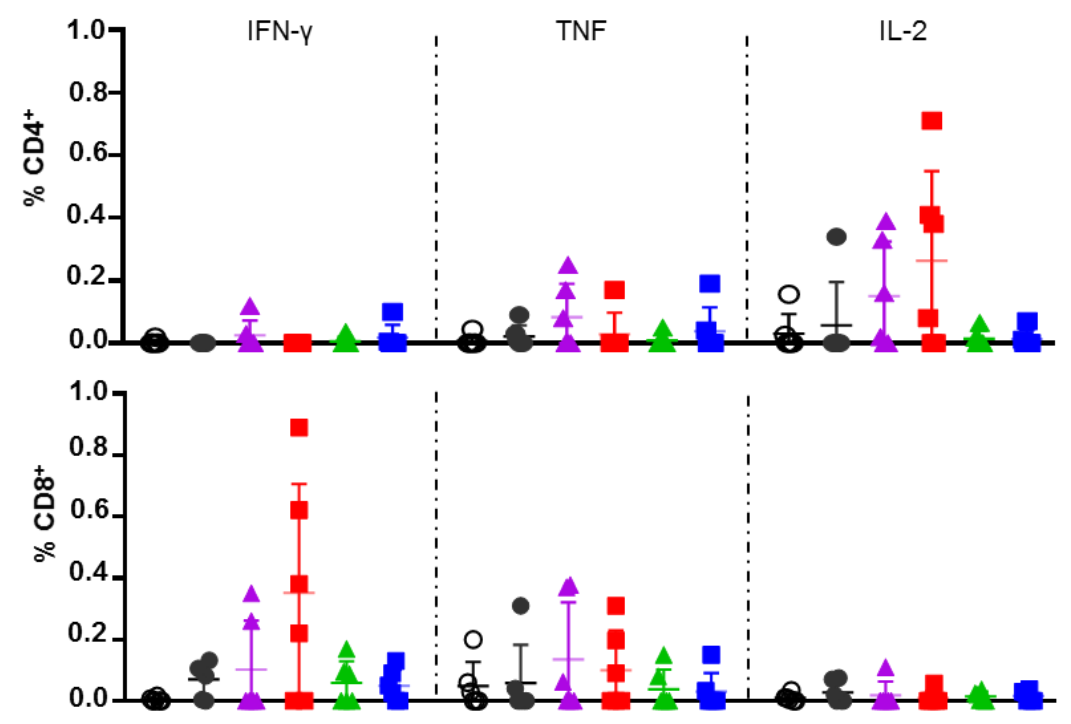

B Y

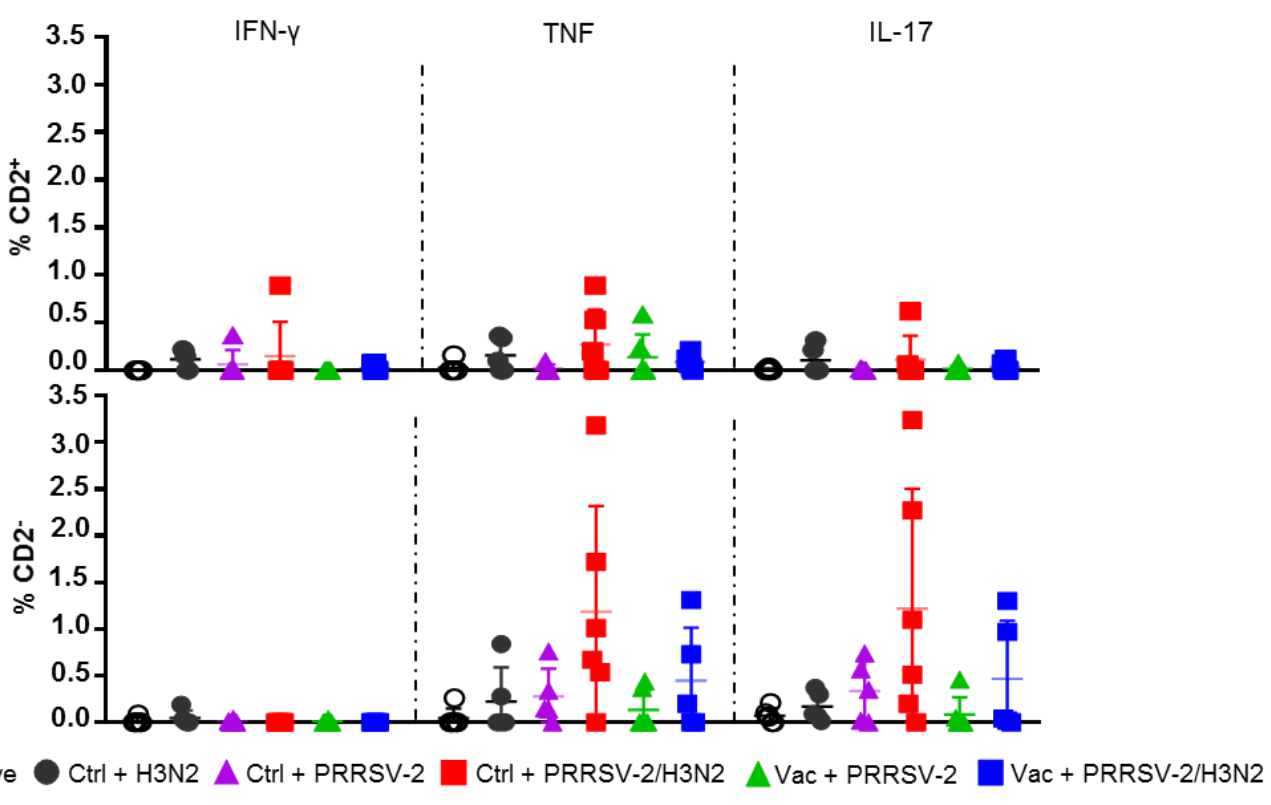

1057 Figure 6: H3N2- specific $\mathbf{T}$ cell responses in bronchoalveolar lavage. Cells

1058 isolated from BALF at $5 \mathrm{dpc}$ were restimulated with swIAV H3N2 (MOI 0.1) or

1059 cultured with medium. Cytokine secretion measured in $\mathrm{CD}^{+}$and $\operatorname{CD}^{+} \beta^{+}(\mathbf{A})$, and

$1060 \mathrm{CD}^{+}$and $\mathrm{CD}^{-} \gamma \delta(\mathbf{B}) \mathrm{T}$ cells are represented. Corrected frequencies of individual

1061 value and the mean $\pm \mathrm{SD}$ are displayed ( $\mathrm{n}=5-6$ per group). Comparisons between 2

1062 groups were made using the Mann-Whitney test. 

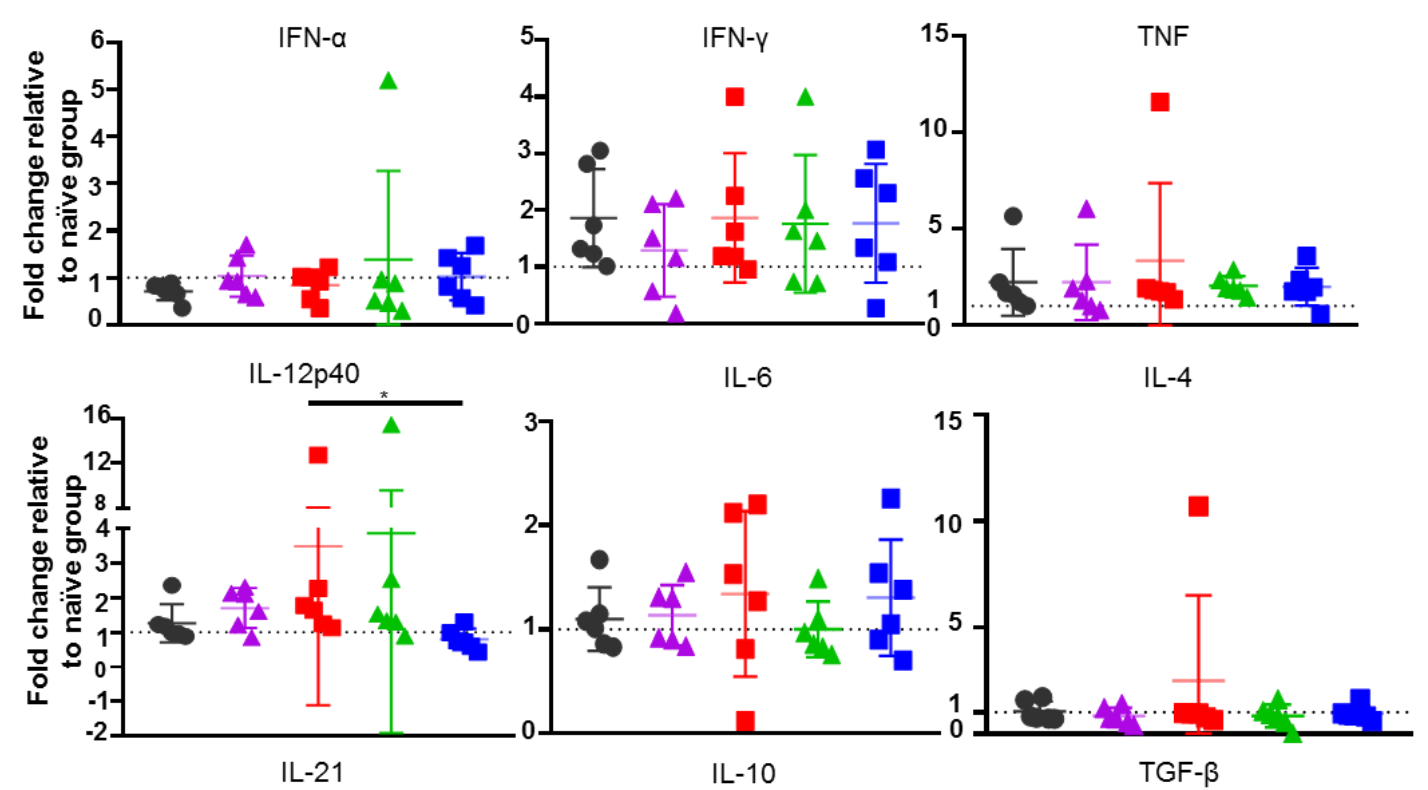

1064
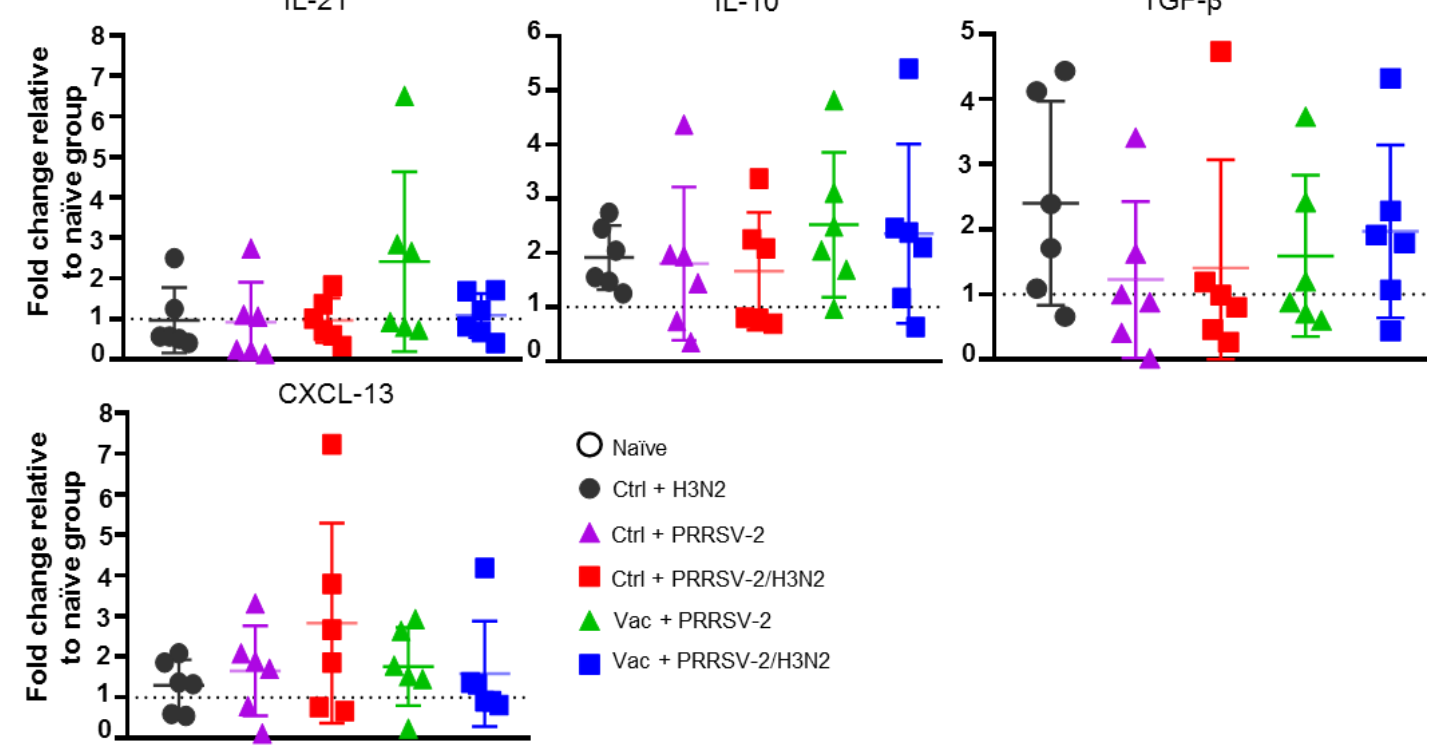

1065 Figure 7: Gene expression in lung tissues. Total RNA was extracted from lung

1066 tissue collected at $5 \mathrm{dpc}$ and the relative mRNA expression of IFN- $\alpha$, IFN- $\gamma$, TNF,

1067 IL-12p40, IL-4, IL-6, IL-21, IL-10, TGF- $\beta$ and CXCL-13 was assessed by qRT-

1068 PCR. Fold changes are shown over naïve group (dash line) after normalization with

1069 GAPDH and RPS24 genes. Individual pig values and the group mean $\pm \mathrm{SD}$ are

1070 displayed ( $\mathrm{n}=5-6$ per group). Comparisons were made using Kruskal-Wallis test and

1071 asterisks indicate significant differences $(* \mathrm{p}<0.05)$. 